\title{
Energy levels, radiative rates, and excitation rates for transitions in $\mathrm{O} \mathrm{iv}^{\star}$
}

\author{
K. M. Aggarwal and F. P. Keenan
}

\begin{abstract}
Astrophysics Research Centre, School of Mathematics and Physics, Queen's University Belfast, Belfast BT7 1NN, Northern Ireland, UK

e-mail: K.Aggarwal@qub.ac.uk
\end{abstract}

Received 26 September 2007 / Accepted 25 May 2008

\begin{abstract}
Aims. In this paper we report calculations for energy levels, radiative rates, and excitation rates for transitions in O IV. Methods. The GRASP (general-purpose relativistic atomic structure package) and FAC (flexible atomic code) were adopted for calculating energy levels and radiative rates, and the Dirac atomic $R$-matrix code (DARC) used to determine the excitation rates.

Results. Oscillator strengths and radiative rates are reported for all E1, E2, M1, and M2 transitions among the lowest 75 levels of O IV. Additionally, lifetimes are reported for all levels and comparisons made with those available in the literature. Finally, effective collision strengths are reported for all transitions over a wide temperature range below $10^{6} \mathrm{~K}$. Comparisons are made with earlier results and the accuracy of the data is assessed.
\end{abstract}

Key words. atomic data - atomic processes

\section{Introduction}

Emission lines of B-like O IV have been widely observed in a variety of astrophysical plasmas. For example, Feldman et al. (1997) observed the spectrum of the solar corona with the Solar Ultraviolet Measurement of Emitted Radiation (SUMER) instrument on board the Solar and Heliospheric Observatory (SOHO), and detected many lines of $\mathrm{O}$ IV over the wavelength range 780-1600 A. Similarly, lines of O IV in the 910-1180 ̊ wavelength range have been detected in late-type stars by Redfield et al. (2002), and in the solar transition region by Pagano et al. (2000). Harper et al. (1999) measured and analysed the O IV lines of the $2 s^{2} 2 p^{2} \mathrm{P}-2 \mathrm{~s} 2 \mathrm{p}^{2}{ }^{4} \mathrm{P}$ multiplet $(\lambda \sim 1400 \AA)$ in the spectra of RR Tel obtained at medium resolution with the Goddard High-Resolution Spectrograph (GHRS) on the Hubble Space Telescope (HST), and demonstrated the density sensitiveness of many line pairs. This was further investigated by Keenan et al. (2002), who analysed many lines of O IV in the spectra of RR Tel as well as the Sun, and demonstrated their usefulness for plasma diagnostics. Finally, Sturm et al. (2002) studied emission lines of $\mathrm{O}$ IV in the infra-red range from active galactic nuclei. However, to reliably analyse observations, atomic data are required for many parameters, such as: energy levels, radiative rates (A-values), and excitation rates or equivalently the effective collision strengths $(\Upsilon)$, which are obtained from the electron impact collision strengths $(\Omega)$. Since experimental values are not available for the desired atomic parameters, except for energy levels, theoretical results are required.

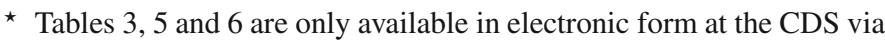
anonymous ftp to cdsarc.u-strasbg.fr $(130.79 .128 .5)$ or via http://cdsweb.u-strasbg.fr/cgi-bin/qcat? J/A+A/486/1053
Considering the importance of O IV many calculations have been performed in the past, as noted by Tayal (2006). However, the most significant calculations, which cover comparatively a larger number of levels and hence transitions, are those of Tachiev \& Froese-Fischer (2000) and Corrégé \& Hibbert (2002, 2004). Tachiev \& Froese-Fischer adopted the multiconfiguration Hartree-Fock (MCHF) program of Froese-Fischer (1991), and reported energy levels, lifetimes and A-values for transitions among the lowest 25 levels of the $2 s^{2} 2 p, 2 s 2 p^{2}$, $2 \mathrm{p}^{3}, 2 \mathrm{~s}^{2} 3 \ell$, and $2 \mathrm{~s} 2 \mathrm{p}\left({ }^{3} \mathrm{P}^{\circ}\right) 3 \mathrm{~s}$ configurations. Similarly, Corrégé \& Hibbert adopted the CIV3 program of Hibbert (1975a) and included extensive CI (configuration interaction) in order to determine the atomic data as accurately as possible, but reported energy levels and A-values for transitions only among the lowest 20 levels of the $2 s^{2} 2 p, 2 s 2 p^{2}, 2 p^{3}$ and $2 s^{2} 3 \ell$ configurations. For the calculations of $\Omega$ and $\Upsilon$, Luo \& Pradhan (1990) and Blum $\&$ Pradhan (1992) adopted the $R$-matrix program of Berrington et al. (1987). They performed the calculations in $L S$ coupling among the 8 states of the $2 s^{2} 2 p, 2 s 2 p^{2}$ and $2 p^{3}$ configurations, and obtained results for fine-structure transitions through algebraic recoupling, which ignores the fine-structure splitting of the terms. The limitations of these calculations have recently been addressed by Tayal (2006), who reported results for energy levels, radiative rates and excitation rates for transitions among the lowest 54 levels of the $2 s^{2} 2 p, 2 s 2 p^{2}, 2 p^{3}, 2 s^{2} 3 \ell, 2 s 2 p 3 \ell, 2 s^{2} 4 s$, and $2 s^{2} 4 p$ configurations of $\mathrm{O} I V$. He also included CI in the generation of wavefunctions adopting the MCHF program. For the calculations of $\Omega$ and subsequently $\Upsilon$, he also used the $R$-matrix program of Berrington et al. (1995). Furthermore, he included one-body relativistic operators in the expansion of the wavefunctions as well as in the scattering process, and resolved resonances in the threshold region in order to account for their contribution to the values of $\Upsilon$. Therefore, Tayal's calculations not only cover 
a wider range of levels/transitions, but also extend the energy and temperature ranges of the data for $\Omega$ and $\Upsilon$ than previously available. However, we see scope for further work for the following reasons.

Firstly, there is a printing error in the listed values of $\Upsilon$ by Tayal (2006) for several transitions, as those (particularly) towards the higher end of the temperature range are larger by up to three orders of magnitude - see, for example, transitions $1-3,32,33 ; 2-3,11,23$ and $5-19,21,24$ in Table 4 of Tayal (2006). We will discuss this point in more detail in Sect. 6. Secondly, and more importantly, there are significant differences between the $\Upsilon$ values of Tayal and Blum \& Pradhan (1992) for several transitions, as shown in his Figs. 7-9. Therefore, an additional calculation may be useful to understand these differences, so that the data may be applied with confidence. Finally, Tayal has reported radiative rates (for electric dipole transitions alone) and excitation rates for only a limited number of transitions, whereas in plasma diagnostics results for all transitions are preferable as demonstrated by Del Zanna et al. (2004), who also emphasized the importance of including the Avalues for all types of transitions, namely electric dipole (E1), electric quadrupole (E2), magnetic dipole (M1), and magnetic quadrupole (M2). Therefore, the aim of the present paper is not only to extend the calculations of Tayal but also to report a complete set of results, which can be confidently applied in plasma modelling.

For our calculations we have adopted the GRASP (generalpurpose relativistic atomic structure package) code to generate the wavefunctions. This code was originally developed as GRASP0 by Grant et al. (1980) and a revised and modified version was published as GRASP1 by Dyall et al. (1989), which has been further updated by Dr. P. H. Norrington. It is a fully relativistic code, and is based on the $j j$ coupling scheme. Further relativistic corrections arising from the Breit interaction and QED effects have also been included. Additionally, we have used the option of extended average level (EAL), in which a weighted (proportional to $2 j+1$ ) trace of the Hamiltonian matrix is minimized. This produces a compromise set of orbitals describing closely lying states with moderate accuracy. Furthermore, in order to assess the accuracy of our results we have performed parallel calculations using the Flexible Atomic Code (FAC) of $\mathrm{Gu}$ (2003), which is available from the website http: // kipac-tree.stanford. edu/fac. This is also a fully relativistic code which provides a variety of atomic parameters, and yields results comparable to GRASP, as already shown for three Mg-like ions by Aggarwal et al. (2007). Thus results from FAC will be helpful in assessing the accuracy of our energy levels and radiative rates.

For the computations of $\Omega$ and subsequently $\Upsilon$, we employ the fully relativistic Dirac atomic R-matrix code (DARC) of P.H. Norrington and I.P. Grant (private communication), as implemented by Ait-Tahar et al. (1996). Furthermore, in our calculations we include all the 68 levels of the $2 \mathrm{~s}^{2} 2 \mathrm{p}, 2 \mathrm{~s} 2 \mathrm{p}^{2}, 2 \mathrm{p}^{3}$, $2 \mathrm{~s}^{2} 3 \ell$, and $2 \mathrm{~s} 2 \mathrm{p} 3 \ell$ configurations, as well as the 7 levels of the $2 s^{2} 4 \ell$ configurations. Thus we are extending the calculations of Tayal (2006) by including an additional 21 levels.

\section{Energy levels}

In Table 1a we list the 75 energy levels belonging to the $2 s^{2} 2 p$, $2 \mathrm{~s} 2 \mathrm{p}^{2}, 2 \mathrm{p}^{3}, 2 \mathrm{~s}^{2} 3 \ell, 2 \mathrm{~s} 2 \mathrm{p} 3 \ell$, and $2 \mathrm{~s}^{2} 4 \ell$ configurations of $\mathrm{O} \mathrm{IV}$. Two sets of results are listed, which have been obtained without and with the inclusion of Breit and QED effects. With the inclusion of these effects, the orderings have slightly changed in two instances, namely for levels 6 and $7\left(2 \mathrm{~s} 2 \mathrm{p}^{2}{ }^{2} \mathrm{D}_{3 / 2,5 / 2}\right)$ and 12 and $13\left(2 \mathrm{p}^{3}{ }^{2} \mathrm{D}_{3 / 2,5 / 2}^{\circ}\right)$. However, their level energies are very close to each other, but the most significant effect is on level 2 $\left(2 \mathrm{~s}^{2} 2 \mathrm{p}^{2} \mathrm{P}_{3 / 2}^{\circ}\right)$ in which case the inclusion of Breit and QED effects has lowered the energy by $15 \%$, and hence brings it closer to the experimental value - see Table 1b. Differences with the experimental energies, compiled by NIST (National Institute of Standards and Technology) and listed at their website http:// physics.nist.gov/PhysRefData, are up to $8 \%$ for the lowest 15 levels, and $\leq 3 \%$ for the remaining higher levels, as shown in Table 1b. This is because the energy levels listed in Table 1a have been obtained by including CI among the levels of the above 13 configurations alone. Since O IV is comparatively a lighter ion $(Z=8)$, CI should be more important than the inclusion of relativistic effects. We discuss this below.

To assess the effect of CI, we have performed a series of calculations by including an increasing number of configurations. However for brevity, we focus only on three, namely: GRASP1, which includes 75 levels of the above listed 13 configurations; GRASP2, which includes an additional 144 levels of the $2 p^{2} 3 \ell$ and $2 p^{2} 4 \ell$ configurations; and GRASP3, which includes a total of 326 levels, the additional 107 levels arising from the $1 \mathrm{~s}^{2} 2 \mathrm{~s} 2 \mathrm{p} 4 \ell, 1 \mathrm{~s} 2 \mathrm{~s}^{2} 2 \mathrm{p}^{2}, 1 \mathrm{~s} 2 \mathrm{~s} 2 \mathrm{p}^{3}$ and $1 \mathrm{~s} 2 \mathrm{p}^{4}$ configurations. The energies obtained, with the inclusion of Breit and QED effects, for the desired 75 levels of Table 1a are listed in Table 1b, along with the experimentally compiled energies of NIST and the earlier theoretical results of Tayal (2006). Furthermore, we have performed parallel calculations by adopting the FAC code of $\mathrm{Gu}$ (2003), although the energy levels obtained from this code are similar to those obtained from GRASP, as also observed earlier for three Mg-like ions (Aggarwal et al. 2007) and many ions of Fe (Aggarwal \& Keenan 2006). Hence these are not included in Table 1b. However, included in Table $1 \mathrm{~b}$ are our results from FAC, which are obtained by including a much larger CI among 686 levels of the 45 configurations, namely $1 \mathrm{~s}^{2} 2 \mathrm{~s}^{2} 2 \mathrm{p}$, $1 \mathrm{~s}^{2} 2 \mathrm{~s} 2 \mathrm{p}^{2}, 1 \mathrm{~s}^{2} 2 \mathrm{p}^{3}, 1 \mathrm{~s} 2 \mathrm{~s}^{2} 2 \mathrm{p}^{2}, 1 \mathrm{~s} 2 \mathrm{~s} 2 \mathrm{p}^{3}, 1 \mathrm{~s} 2 \mathrm{p}^{4}, 1 \mathrm{~s}^{2} 2 \mathrm{~s}^{2} 3 \ell, 1 \mathrm{~s}^{2} 2 \mathrm{p}^{2} 3 \ell$, $1 \mathrm{~s}^{2} 2 \mathrm{~s} 2 \mathrm{p} 3 \ell, 1 \mathrm{~s}^{2} 2 \mathrm{~s}^{2} 4 \ell, 1 \mathrm{~s}^{2} 2 \mathrm{p}^{2} 4 \ell, 1 \mathrm{~s}^{2} 2 \mathrm{~s} 2 \mathrm{p} 4 \ell, 2 \mathrm{~s} 2 \mathrm{p} 5 \ell, 2 \mathrm{~s} 2 \mathrm{p} 6 \ell$, and $2 \mathrm{~s} 2 \mathrm{p} 7 \ell$. This inclusion of a larger CI will help us in further assessing the accuracy of our energy levels.

As stated earlier, the energy levels obtained without external CI (GRASP1) are not in satisfactory agreement with the corresponding experimental results, and differ in magnitude by up to $8 \%$. More importantly, the level orderings are different in a few instances, in particular for the $2 s^{2} 4 s^{2} S_{1 / 2}$ level. Inclusion of CI with external orbitals (and configurations), as in GRASP2, improves the energies significantly (by up to 5\%) for many levels, such as 16-20 and 55-58. Differences with the experimental values are now less than $6 \%$ for the lowest 15 levels and less than $2 \%$ for the higher ones. Moreover, the level orderings are now in comparatively better agreement, although some differences still remain. A further inclusion of CI, as in GRASP3, makes an insignificant effect $(\leq 1 \%)$, and differences with the experimental values as well as the level orderings remain (nearly) the same. Therefore, it may be fair to state that the further addition of CI than included in GRASP2 is of no appreciable advantage as far as the 75 levels of Tables $1 \mathrm{a}$ and $\mathrm{b}$ are concerned. This is further confirmed by the results obtained from FAC, which includes more extensive CI than in the GRASP calculations. In fact, the energy for level $2\left(2 s^{2} 2 p^{2} \mathrm{P}_{3 / 2}^{\circ}\right)$ has become worse as it is now lower than the experimental one by $\sim 10 \%$, whereas the differences for other levels (up to 15) still remain around 5\%. Therefore, in conclusion we may state that our energy levels obtained from the GRASP2 calculations are as good as those obtained with a larger 
Table 1. a) Energy levels (in Ryd) of O IV.

\begin{tabular}{|c|c|c|c|c|c|c|c|c|c|}
\hline Index & Configuratio & Level & "GRASP1a & GRASP1b & Index & Configuratio & 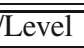 & "GRASP1a & GRASP1b \\
\hline 1 & $2 s^{2} 2 p$ & ${ }^{2} \mathrm{P}_{1 / 2}^{\circ}$ & 0.00000 & 0.00000 & 39 & $2 \mathrm{~s} 2 \mathrm{p} 3 \mathrm{~d}$ & ${ }^{4} \mathrm{~F}_{5 / 2}^{\circ}$ & 4.46062 & 4.45946 \\
\hline 2 & $2 s^{2} 2 p$ & ${ }^{2} \mathrm{P}_{3 / 2}^{\circ}$ & 0.00391 & 0.00340 & 40 & $2 \mathrm{~s} 2 \mathrm{p} 3 \mathrm{~d}$ & ${ }^{4} \mathrm{~F}_{7 / 2}^{\circ}$ & 4.46187 & 4.46049 \\
\hline 3 & $2 \mathrm{~s} 2 \mathrm{p}^{2}$ & ${ }^{4} \mathrm{P}_{1 / 2}$ & 0.61689 & 0.61674 & 41 & $2 \mathrm{~s} 2 \mathrm{p} 3 \mathrm{~d}$ & ${ }^{4} \mathrm{~F}_{9 / 2}^{\circ}$ & 4.46351 & 4.46189 \\
\hline 4 & $2 \mathrm{~s} 2 \mathrm{p}^{2}$ & ${ }^{4} \mathrm{P}_{3 / 2}$ & 0.61820 & 0.61789 & 42 & $2 \mathrm{~s} 2 \mathrm{p}\left({ }^{3} \mathrm{P}^{\circ}\right) 3 \mathrm{p}$ & ${ }^{2} \mathrm{~S}_{1 / 2}$ & 4.46955 & 4.46847 \\
\hline 5 & $2 \mathrm{~s} 2 \mathrm{p}^{2}$ & ${ }^{4} \mathrm{P}_{5 / 2}$ & 0.62036 & 0.61945 & 43 & $2 \mathrm{~s} 2 \mathrm{p} 3 \mathrm{~d}$ & ${ }^{4} \mathrm{D}_{1 / 2}^{\circ}$ & 4.50938 & 4.50812 \\
\hline 6 & $2 \mathrm{~s} 2 \mathrm{p}^{2}$ & ${ }^{2} \mathrm{D}_{3 / 2}$ & 1.22619 & 1.22565 & 44 & $2 \mathrm{~s} 2 \mathrm{p} 3 \mathrm{~d}$ & ${ }^{4} \mathrm{D}_{3 / 2}^{\mathrm{o}}$ & 4.50963 & 4.50836 \\
\hline 7 & $2 \mathrm{~s} 2 \mathrm{p}^{2}$ & ${ }^{2} \mathrm{D}_{5 / 2}$ & 1.22620 & 1.22550 & 45 & $2 \mathrm{~s} 2 \mathrm{p} 3 \mathrm{~d}$ & ${ }^{4} D_{5 / 2}^{0 / 2}$ & 4.51010 & 4.50876 \\
\hline 8 & $2 \mathrm{~s} 2 \mathrm{p}^{2}$ & ${ }^{2} \mathrm{~S}_{1 / 2}$ & 1.56688 & 1.56661 & 46 & $2 \mathrm{~s} 2 \mathrm{p} 3 \mathrm{~d}$ & ${ }^{4} \mathrm{D}_{7 / 2}^{\mathrm{s} / 2}$ & 4.51086 & 4.50935 \\
\hline 9 & $2 \mathrm{~s} 2 \mathrm{p}^{2}$ & ${ }^{2} \mathrm{P}_{1 / 2}$ & 1.79627 & 1.79611 & 47 & $2 \mathrm{~s} 2 \mathrm{p} 3 \mathrm{~d}$ & ${ }^{4} \mathrm{P}_{5 / 2}^{\mathrm{o} / 2}$ & 4.53538 & 4.53415 \\
\hline 10 & $2 \mathrm{~s} 2 \mathrm{p}^{2}$ & ${ }^{2} \mathrm{P}_{3 / 2}$ & 1.79888 & 1.79821 & 48 & $2 \mathrm{~s} 2 \mathrm{p}\left({ }^{3} \mathrm{P}^{\circ}\right) 3 \mathrm{~d}$ & ${ }^{2} \mathrm{D}_{3 / 2}^{\circ}$ & 4.53587 & 4.53461 \\
\hline 11 & $2 \mathrm{p}^{3}$ & ${ }^{4} S_{3 / 2}^{\circ}$ & 2.13306 & 2.13235 & 49 & $2 \mathrm{~s} 2 \mathrm{p}\left({ }^{3} \mathrm{P}^{\circ}\right) 3 \mathrm{~d}$ & ${ }^{2} \mathrm{D}_{5 / 2}^{0 / 2}$ & 4.53741 & 4.53591 \\
\hline 12 & $2 p^{3}$ & ${ }^{2} \mathrm{D}_{3 / 2}^{\circ}$ & 2.45173 & 2.45115 & 50 & $2 \mathrm{~s} 2 \mathrm{p} 3 \mathrm{~d}$ & ${ }^{4} \mathrm{P}_{3 / 2}^{\circ}$ & 4.53744 & 4.53601 \\
\hline 13 & $2 p^{3}$ & ${ }^{2} \mathrm{D}_{5 / 2}^{\circ}$ & 2.45177 & 2.45079 & 51 & $2 \mathrm{~s} 2 \mathrm{p} 3 \mathrm{~d}$ & ${ }^{4} \mathrm{P}_{1 / 2}^{\circ}$ & 4.53804 & 4.53659 \\
\hline 14 & $2 p^{3}$ & ${ }^{2} \mathrm{P}_{1 / 2}^{\mathrm{o} / 2}$ & 2.79607 & 2.79564 & 52 & $2 s^{2} 4 s$ & ${ }^{2} \mathrm{~S}_{1 / 2}^{1 / 2}$ & 4.55019 & 4.54922 \\
\hline 15 & $2 \mathrm{p}^{3}$ & ${ }^{2} \mathrm{P}_{3 / 2}^{\circ}$ & 2.79626 & 2.79572 & 53 & $2 \mathrm{~s} 2 \mathrm{p}\left({ }^{3} \mathrm{P}^{\circ}\right) 3 \mathrm{~d}$ & ${ }^{2} \mathrm{~F}_{5 / 2}^{\circ}$ & 4.62727 & 4.62610 \\
\hline 16 & $2 s^{2} 3 s$ & ${ }^{2} \mathrm{~S}_{1 / 2}^{3 / 2}$ & 3.35665 & 3.35580 & 54 & $2 \mathrm{~s} 2 \mathrm{p}\left({ }^{3} \mathrm{P}^{\circ}\right) 3 \mathrm{~d}$ & ${ }^{2} \mathrm{~F}_{7 / 2}^{\circ / 2}$ & 4.62991 & 4.62832 \\
\hline 17 & $2 s^{2} 3 p$ & ${ }^{2} \mathrm{P}_{1 / 2}^{\circ}$ & 3.65598 & 3.65517 & 55 & $2 \mathrm{~s} 2 \mathrm{p}\left({ }^{3} \mathrm{P}^{\circ}\right) 3 \mathrm{~d}$ & ${ }^{2} \mathrm{P}_{3 / 2}^{\circ}$ & 4.65788 & 4.65671 \\
\hline 18 & $2 s^{2} 3 p$ & ${ }^{2} \mathrm{P}_{3 / 2}^{\circ}$ & 3.65686 & 3.65594 & 56 & $2 \mathrm{~s} 2 \mathrm{p}\left({ }^{3} \mathrm{P}^{\circ}\right) 3 \mathrm{~d}$ & ${ }^{2} \mathrm{P}_{1 / 2}^{\mathrm{s} / 2}$ & 4.65851 & 4.65732 \\
\hline 19 & $2 s^{2} 3 d$ & ${ }^{2} \mathrm{D}_{3 / 2}^{3 / 2}$ & 3.94327 & 3.94227 & 57 & $2 s^{2} 4 p$ & ${ }^{2} \mathrm{P}_{3 / 2}^{1 / 2}$ & 4.67029 & 4.66919 \\
\hline 20 & $2 s^{2} 3 d$ & ${ }^{2} \mathrm{D}_{5 / 2}$ & 3.94347 & 3.94245 & 58 & $2 s^{2} 4 p$ & ${ }^{2} \mathrm{P}_{1 / 2}^{\mathrm{o} / 2}$ & 4.67085 & 4.66966 \\
\hline 21 & $2 \mathrm{~s} 2 \mathrm{p} 3 \mathrm{~s}$ & ${ }^{4} \mathrm{P}_{1 / 2}^{\circ}$ & 3.94962 & 3.94881 & 59 & $2 s^{2} 4 d$ & ${ }^{2} \mathrm{D}_{3 / 2}$ & 4.76352 & 4.76252 \\
\hline 22 & $2 \mathrm{~s} 2 \mathrm{p} 3 \mathrm{~s}$ & ${ }^{4} \mathrm{P}_{3 / 2}^{\circ}$ & 3.95116 & 3.95005 & 60 & $2 s^{2} 4 d$ & ${ }^{2} \mathrm{D}_{5 / 2}$ & 4.76358 & 4.76257 \\
\hline 23 & $2 \mathrm{~s} 2 \mathrm{p} 3 \mathrm{~s}$ & ${ }^{4} \mathrm{P}_{5 / 2}^{\mathrm{og}}$ & 3.95375 & 3.95231 & 61 & $2 s^{2} 4 f$ & ${ }^{2} \mathrm{~F}_{5 / 2}^{\circ}$ & 4.77634 & 4.77532 \\
\hline 24 & $2 \mathrm{~s} 2 \mathrm{p}\left({ }^{3} \mathrm{P}^{\circ}\right) 3 \mathrm{~s}$ & ${ }^{2} \mathrm{P}_{1 / 2}^{\mathrm{o} / 2}$ & 4.13075 & 4.12994 & 62 & $2 s^{2} 4 f$ & ${ }^{2} \mathrm{~F}_{7 / 2}^{\circ}$ & 4.77637 & 4.77535 \\
\hline 25 & $2 \mathrm{~s} 2 \mathrm{p}\left({ }^{3} \mathrm{P}^{\circ}\right) 3 \mathrm{~s}$ & ${ }^{2} \mathrm{P}_{3 / 2}^{\circ}$ & 4.13351 & 4.13230 & 63 & $2 \mathrm{~s} 2 \mathrm{p}\left({ }^{1} \mathrm{P}^{\circ}\right) 3 \mathrm{~s}$ & ${ }^{2} \mathrm{P}_{1 / 2}^{\circ}$ & 4.81607 & 4.81499 \\
\hline 26 & $2 \mathrm{~s} 2 \mathrm{p} 3 \mathrm{p}$ & ${ }^{4} D_{1 / 2}$ & 4.21678 & 4.21596 & 64 & $2 \mathrm{~s} 2 \mathrm{p}\left({ }^{1} \mathrm{P}^{\circ}\right) 3 \mathrm{~s}$ & ${ }^{2} \mathrm{P}_{3 / 2}^{\mathrm{o} / \mathrm{L}}$ & 4.81629 & 4.81517 \\
\hline 27 & $2 \mathrm{~s} 2 \mathrm{p} 3 \mathrm{p}$ & ${ }^{4} \mathrm{D}_{3 / 2}$ & 4.21773 & 4.21673 & 65 & $2 \mathrm{~s} 2 \mathrm{p}\left({ }^{1} \mathrm{P}^{\circ}\right) 3 \mathrm{p}$ & ${ }^{2} \mathrm{D}_{3 / 2}$ & 5.08458 & 5.08345 \\
\hline 28 & $2 \mathrm{~s} 2 \mathrm{p} 3 \mathrm{p}$ & ${ }^{4} D_{5 / 2}$ & 4.21929 & 4.21805 & 66 & $2 \mathrm{~s} 2 \mathrm{p}\left({ }^{1} \mathrm{P}^{\circ}\right) 3 \mathrm{p}$ & ${ }^{2} \mathrm{D}_{5 / 2}$ & 5.08496 & 5.08377 \\
\hline 29 & $2 \mathrm{~s} 2 \mathrm{p} 3 \mathrm{p}$ & ${ }^{4} \mathrm{D}_{7 / 2}$ & 4.22148 & 4.21996 & 67 & $2 \mathrm{~s} 2 \mathrm{p}\left({ }^{1} \mathrm{P}^{\circ}\right) 3 \mathrm{p}$ & ${ }^{2} \mathrm{P}_{1 / 2}$ & 5.10430 & 5.10329 \\
\hline 30 & $2 \mathrm{~s} 2 \mathrm{p}\left({ }^{3} \mathrm{P}^{\circ}\right) 3 \mathrm{p}$ & ${ }^{2} \mathrm{P}_{1 / 2}$ & 4.25507 & 4.25396 & 68 & $2 \mathrm{~s} 2 \mathrm{p}\left({ }^{1} \mathrm{P}^{\circ}\right) 3 \mathrm{p}$ & ${ }^{2} \mathrm{P}_{3 / 2}$ & 5.10497 & 5.10382 \\
\hline 31 & $2 \mathrm{~s} 2 \mathrm{p}\left({ }^{3} \mathrm{P}^{\circ}\right) 3 \mathrm{p}$ & ${ }^{2} \mathrm{P}_{3 / 2}$ & 4.25594 & 4.25469 & 69 & $2 \mathrm{~s} 2 \mathrm{p}\left({ }^{1} \mathrm{P}^{\circ}\right) 3 \mathrm{p}$ & ${ }^{2} \mathrm{~S}_{1 / 2}$ & 5.19129 & 5.19023 \\
\hline 32 & $2 \mathrm{~s} 2 \mathrm{p} 3 \mathrm{p}$ & ${ }^{4} S_{3 / 2}$ & 4.26211 & 4.26082 & 70 & $2 \mathrm{~s} 2 \mathrm{p}\left({ }^{1} \mathrm{P}^{\circ}\right) 3 \mathrm{~d}$ & ${ }^{2} \mathrm{D}_{3 / 2}^{\circ}$ & 5.32095 & 5.31967 \\
\hline 33 & $2 \mathrm{~s} 2 \mathrm{p} 3 \mathrm{p}$ & ${ }^{4} \mathrm{P}_{1 / 2}$ & 4.31305 & 4.31209 & 71 & $2 \mathrm{~s} 2 \mathrm{p}\left({ }^{1} \mathrm{P}^{\circ}\right) 3 \mathrm{~d}$ & ${ }^{2} \mathrm{D}_{5 / 2}^{\mathrm{og}}$ & 5.32120 & 5.31989 \\
\hline 34 & $2 \mathrm{~s} 2 \mathrm{p} 3 \mathrm{p}$ & ${ }^{4} \mathrm{P}_{3 / 2}$ & 4.31400 & 4.31293 & 72 & $2 \mathrm{~s} 2 \mathrm{p}\left({ }^{1} \mathrm{P}^{\circ}\right) 3 \mathrm{~d}$ & ${ }^{2} \mathrm{~F}_{7 / 2}^{0}$ & 5.33156 & 5.33027 \\
\hline 35 & $2 \mathrm{~s} 2 \mathrm{p} 3 \mathrm{p}$ & ${ }^{4} \mathrm{P}_{5 / 2}$ & 4.31544 & 4.31408 & 73 & $2 \mathrm{~s} 2 \mathrm{p}\left({ }^{1} \mathrm{P}^{\circ}\right) 3 \mathrm{~d}$ & ${ }^{2} \mathrm{~F}_{5 / 2}^{\circ}$ & 5.33173 & 5.33043 \\
\hline 36 & $2 \mathrm{~s} 2 \mathrm{p}\left({ }^{3} \mathrm{P}^{\circ}\right) 3 \mathrm{p}$ & ${ }^{2} \mathrm{D}_{3 / 2}$ & 4.38030 & 4.37931 & 74 & $2 \mathrm{~s} 2 \mathrm{p}\left({ }^{1} \mathrm{P}^{\circ}\right) 3 \mathrm{~d}$ & ${ }^{2} \mathrm{P}_{1 / 2}^{\mathrm{o}}$ & 5.40706 & 5.40584 \\
\hline 37 & $2 \mathrm{~s} 2 \mathrm{p}\left({ }^{3} \mathrm{P}^{\circ}\right) 3 \mathrm{p}$ & ${ }^{2} \mathrm{D}_{5 / 2}$ & 4.38294 & 4.38158 & 75 & $2 \mathrm{~s} 2 \mathrm{p}\left({ }^{1} \mathrm{P}^{\circ}\right) 3 \mathrm{~d}$ & ${ }^{2} \mathrm{P}_{3 / 2}^{\mathrm{o} / 2}$ & 5.40718 & 5.40592 \\
\hline 38 & $2 \mathrm{~s} 2 \mathrm{p} 3 \mathrm{~d}$ & ${ }^{4} \mathrm{~F}_{3 / 2}^{\circ}$ & 4.45975 & 4.45877 & & & & & \\
\hline
\end{tabular}

GRASP1a: energies from the GRASP code without Breit and QED effects from 75 level calculations.

GRASP1b: energies from the GRASP code with Breit and QED effects from 75 level calculations.

CI in the GRASP3 and FAC calculations. Similarly, the energy levels of Tayal (2006) obtained from the MCHF code also differ from the experimental results by $5 \%$ for level $2\left(2 \mathrm{~s}^{2} 2 \mathrm{p}^{2} \mathrm{P}_{3 / 2}^{\circ}\right)$, and less than $3 \%$ for the remaining levels. In a few instances, such as for levels $6 / 7\left(2 \mathrm{~s} 2 \mathrm{p}^{2}{ }^{2} \mathrm{D}_{3 / 2,5 / 2}\right), 12 / 13\left(2 \mathrm{p}^{3}{ }^{2} \mathrm{D}_{3 / 2,5 / 2}^{\circ}\right)$ and $19 / 20\left(2 s^{2} 3 d^{2} D_{3 / 2,5 / 2}\right)$, his level orderings are different from the experimental or our theoretical results, but energy differences between these levels are very small. However, two of his levels $\left(2 \mathrm{~s} 2 \mathrm{p} 3 \mathrm{~d}^{4} \mathrm{~F}_{3 / 2,5 / 2}^{\circ}\right)$ are non-degenerate in energy, whereas the level $2 \mathrm{~s} 2 \mathrm{p}\left({ }^{3} \mathrm{P}^{\circ}\right) 3 \mathrm{~d}{ }^{2} \mathrm{P}_{1 / 2}^{\circ}(62)$ is clearly misplaced (apart from having the same energy as for the level $2 \mathrm{~s} 2 \mathrm{p} 3 \mathrm{~d}{ }^{4} \mathrm{D}_{1 / 2}^{\circ}$ ), and the energy for the level $44\left(2 s^{2} 4 p^{2} \mathrm{P}_{1 / 2}^{\circ}\right)$ is missing. This may be perhaps due to some printing error.

Our calculations for energy levels with differing amount of CI and from two independent codes (GRASP and FAC) provide consistent results, as shown in Table $1 \mathrm{~b}$ and discussed above. However, some differences with the experimental results in the orderings remain, such as for levels 43-45 and 59-60. Sometimes the levels of the same $J$ value, either from the same or different configuration(s), are highly mixed (see, for example, Aggarwal \& Keenan 2006), which makes it difficult to identify these unambiguously. However, we would like to emphasize here that this is not the case for O IV, as all the levels listed in Table 1 have dominant eigenvectors in all sets of calculations performed. Nevertheless, most of the (misplaced) levels are close to one another in energy, and differing amount of CI produce different orderings as seen from Table $1 \mathrm{~b}$. Our final orderings are those listed in Table $1 \mathrm{~b}$, but perhaps scope remains for further improvements. To our knowledge, no other theoretical results are available in the literature with which to compare, particularly for the higher levels. However, results for the lowest 25 levels are available from the MCHF calculations of Tachiev $\&$ Froese-Fischer (2000), and for the lowest 20 levels from the 
Table 1. b) Comparison of energy levels (in Ryd) of O IV and their lifetimes ( $\tau$ in s). $a \pm b \equiv a \times 10^{ \pm b}$.

\begin{tabular}{|c|c|c|c|c|c|c|c|c|c|}
\hline Index & Configuration & Level & NIST & GRASP1 & GRASP2 & "GRASP3 & $\overline{\text { FAC }}$ & MCHF & $\tau$ \\
\hline 1 & $2 s^{2} 2 p$ & ${ }^{2} \mathrm{P}_{1 / 2}^{\circ}$ & 0.00000 & 0.00000 & 0.00000 & 0.00000 & 0.00000 & 0.00000 & $\ldots$ \\
\hline 2 & $2 s^{2} 2 p$ & ${ }^{2} \mathrm{P}_{3 / 2}^{\circ}$ & 0.00352 & 0.00340 & 0.00339 & 0.00333 & 0.00321 & 0.00337 & $2.168+03$ \\
\hline 3 & $2 \mathrm{~s} 2 \mathrm{p}^{2}$ & ${ }^{4} \mathrm{P}_{1 / 2}$ & 0.65101 & 0.61674 & 0.61591 & 0.62126 & 0.63506 & 0.65294 & $5.132-04$ \\
\hline 4 & $2 \mathrm{~s} 2 \mathrm{p}^{2}$ & ${ }^{4} \mathrm{P}_{3 / 2}$ & 0.65220 & 0.61789 & 0.61705 & 0.62238 & 0.63612 & 0.65405 & $5.985-03$ \\
\hline 5 & $2 \mathrm{~s} 2 \mathrm{p}^{2}$ & ${ }^{4} \mathrm{P}_{5 / 2}$ & 0.65388 & 0.61945 & 0.61860 & 0.62389 & 0.63754 & 0.65590 & $1.528-03$ \\
\hline 6 & $2 \mathrm{~s} 2 \mathrm{p}^{2}$ & ${ }^{2} \mathrm{D}_{5 / 2}$ & 1.15673 & 1.22550 & 1.20760 & 1.20923 & 1.21392 & 1.17890 & $1.208-09$ \\
\hline 7 & $2 \mathrm{~s} 2 \mathrm{p}^{2}$ & ${ }^{2} \mathrm{D}_{3 / 2}$ & 1.15686 & 1.22565 & 1.20775 & 1.20938 & 1.21409 & 1.17885 & 1.197-09 \\
\hline 8 & $2 \mathrm{~s} 2 \mathrm{p}^{2}$ & ${ }^{2} \mathrm{~S}_{1 / 2}$ & 1.49782 & 1.56661 & 1.56308 & 1.55977 & 1.55948 & 1.53836 & $2.200-10$ \\
\hline 9 & $2 \mathrm{~s} 2 \mathrm{p}^{2}$ & ${ }^{2} \mathrm{P}_{1 / 2}$ & 1.64466 & 1.79611 & 1.73160 & 1.73024 & 1.72950 & 1.68077 & $1.206-10$ \\
\hline 10 & $2 \mathrm{~s} 2 \mathrm{p}^{2}$ & ${ }^{2} \mathrm{P}_{3 / 2}$ & 1.64688 & 1.79821 & 1.73369 & 1.73230 & 1.73148 & 1.68289 & $1.205-10$ \\
\hline 11 & $2 \mathrm{p}^{3}$ & ${ }^{4} \mathrm{~S}_{3 / 2}^{\circ}$ & 2.10993 & 2.13235 & 2.11408 & 2.11925 & 2.13476 & 2.11881 & $1.425-10$ \\
\hline 12 & $2 \mathrm{p}^{3}$ & ${ }^{2} \mathrm{D}_{5 / 2}^{\circ}$ & 2.32515 & 2.45079 & 2.41448 & 2.41263 & 2.41731 & 2.35208 & $5.076-10$ \\
\hline 13 & $2 \mathrm{p}^{3}$ & ${ }^{2} \mathrm{D}_{3 / 2}^{\circ}$ & 2.32542 & 2.45115 & 2.41481 & 2.41295 & 2.41763 & 2.35181 & $5.082-10$ \\
\hline 14 & $2 \mathrm{p}^{3}$ & ${ }^{2} \mathrm{P}_{1 / 2}^{\circ}$ & 2.63370 & 2.79564 & 2.74605 & 2.74490 & 2.74770 & 2.67691 & $1.805-10$ \\
\hline 15 & $2 p^{3}$ & ${ }^{2} \mathrm{P}_{3 / 2}^{\circ}$ & 2.63378 & 2.79572 & 2.74613 & 2.74497 & 2.74775 & 2.67701 & $1.808-10$ \\
\hline 16 & $2 s^{2} 3 s$ & ${ }^{2} \mathrm{~S}_{1 / 2}$ & 3.25882 & 3.35580 & 3.18766 & 3.17805 & 3.20753 & 3.25903 & $1.218-10$ \\
\hline 17 & $2 s^{2} 3 p$ & ${ }^{2} \mathrm{P}_{1 / 2}^{\circ}$ & 3.55541 & 3.65517 & 3.48283 & 3.47584 & 3.51651 & 3.54775 & $9.034-10$ \\
\hline 18 & $2 s^{2} 3 p$ & ${ }^{2} \mathrm{P}_{3 / 2}^{\mathrm{o}}$ & 3.55620 & 3.65594 & 3.48362 & 3.47663 & 3.51716 & 3.55647 & $9.030-10$ \\
\hline 19 & $2 s^{2} 3 d$ & ${ }^{2} \mathrm{D}_{3 / 2}$ & 3.82307 & 3.94227 & 3.75789 & 3.74795 & 3.79136 & 3.83720 & $2.862-11$ \\
\hline 20 & $2 s^{2} 3 d$ & ${ }^{2} \mathrm{D}_{5 / 2}$ & 3.82323 & 3.94245 & 3.75803 & 3.74809 & 3.79148 & 3.83706 & $2.866-11$ \\
\hline 21 & $2 \mathrm{~s} 2 \mathrm{p} 3 \mathrm{~s}$ & ${ }^{4} \mathrm{P}_{1 / 2}^{\circ}$ & 3.99909 & 3.94881 & 3.92915 & 3.92182 & 3.96157 & 4.00397 & $9.425-11$ \\
\hline 22 & $2 \mathrm{~s} 2 \mathrm{p} 3 \mathrm{~s}$ & ${ }^{4} \mathrm{P}_{3 / 2}^{\mathrm{o}}$ & 4.00032 & 3.95005 & 3.93038 & 3.92303 & 3.96270 & 4.00509 & $9.420-11$ \\
\hline 23 & $2 \mathrm{~s} 2 \mathrm{p} 3 \mathrm{~s}$ & ${ }^{4} \mathrm{P}_{5 / 2}^{\circ}$ & 4.00257 & 3.95231 & 3.93261 & 3.92524 & 3.96476 & 4.00712 & $9.408-11$ \\
\hline 24 & $2 \mathrm{~s} 2 \mathrm{p}\left({ }^{3} \mathrm{P}^{\circ}\right) 3 \mathrm{~s}$ & ${ }^{2} \mathrm{P}_{1 / 2}^{\mathrm{o}}$ & 4.12628 & 4.12994 & 4.07957 & 4.07207 & 4.12550 & 4.14020 & $1.127-10$ \\
\hline 25 & $2 \mathrm{~s} 2 \mathrm{p}\left({ }^{3} \mathrm{P}^{\circ}\right) 3 \mathrm{~s}$ & ${ }^{2} \mathrm{P}_{3 / 2}^{\circ / 2}$ & 4.12869 & 4.13230 & 4.08194 & 4.07441 & 4.12773 & 4.14239 & $1.122-10$ \\
\hline 26 & $2 \mathrm{~s} 2 \mathrm{p}\left({ }^{3} \mathrm{P}^{\circ}\right) 3 \mathrm{p}$ & ${ }^{2} \mathrm{P}_{1 / 2}$ & 4.25771 & 4.25396 & 4.19583 & 4.18886 & 4.23302 & 4.26653 & $6.806-11$ \\
\hline 27 & $2 \mathrm{~s} 2 \mathrm{p}\left({ }^{3} \mathrm{P}^{\circ}\right) 3 \mathrm{p}$ & ${ }^{2} \mathrm{P}_{3 / 2}$ & 4.25876 & 4.25469 & 4.19691 & 4.18994 & 4.23394 & 4.26749 & $6.733-11$ \\
\hline 28 & $2 \mathrm{~s} 2 \mathrm{p} 3 \mathrm{p}$ & ${ }^{4} \mathrm{D}_{1 / 2}$ & 4.26780 & 4.21596 & 4.20150 & 4.19353 & 4.23581 & 4.27826 & 1.594-09 \\
\hline 29 & $2 \mathrm{~s} 2 \mathrm{p} 3 \mathrm{p}$ & ${ }^{4} \mathrm{D}_{3 / 2}$ & 4.26851 & 4.21673 & 4.20218 & 4.19419 & 4.23609 & 4.27890 & $2.202-09$ \\
\hline 30 & $2 \mathrm{~s} 2 \mathrm{p} 3 \mathrm{p}$ & ${ }^{4} \mathrm{D}_{5 / 2}$ & 4.26975 & 4.21805 & 4.20336 & 4.19533 & 4.23686 & 4.28001 & 8.692-09 \\
\hline 31 & $2 \mathrm{~s} 2 \mathrm{p} 3 \mathrm{p}$ & ${ }^{4} \mathrm{D}_{7 / 2}$ & 4.27166 & 4.21996 & 4.20526 & 4.19721 & 4.23892 & 4.28170 & $8.662-09$ \\
\hline 32 & $2 \mathrm{~s} 2 \mathrm{p} 3 \mathrm{p}$ & ${ }^{4} S_{3 / 2}$ & 4.32376 & 4.26082 & 4.25326 & 4.24610 & 4.28997 & 4.33524 & 5.477-09 \\
\hline 33 & $2 \mathrm{~s} 2 \mathrm{p} 3 \mathrm{p}$ & ${ }^{4} \mathrm{P}_{1 / 2}$ & 4.36359 & 4.31209 & 4.29685 & 4.29231 & 4.36063 & 4.37240 & $3.409-09$ \\
\hline 34 & $2 \mathrm{~s} 2 \mathrm{p} 3 \mathrm{p}$ & ${ }^{4} \mathrm{P}_{3 / 2}$ & 4.36445 & 4.31293 & 4.29769 & 4.29314 & 4.36088 & 4.37323 & $3.405-09$ \\
\hline 35 & $2 \mathrm{~s} 2 \mathrm{p} 3 \mathrm{p}$ & ${ }^{4} \mathrm{P}_{5 / 2}$ & 4.36563 & 4.31408 & 4.29882 & 4.29426 & 4.36221 & 4.37433 & $3.388-09$ \\
\hline 36 & $2 s^{2} 4 s$ & ${ }^{2} \mathrm{~S}_{1 / 2}$ & 4.42713 & 4.54922 & 4.34934 & 4.33932 & 4.37941 & 4.41291 & $2.991-10$ \\
\hline 37 & $2 \mathrm{~s} 2 \mathrm{p}\left({ }^{3} \mathrm{P}^{\circ}\right) 3 \mathrm{p}$ & ${ }^{2} \mathrm{D}_{3 / 2}$ & 4.39838 & 4.37931 & 4.35552 & 4.34694 & 4.40473 & 4.41476 & $6.850-11$ \\
\hline 38 & $2 \mathrm{~s} 2 \mathrm{p}\left({ }^{3} \mathrm{P}^{\circ}\right) 3 \mathrm{p}$ & ${ }^{2} \mathrm{D}_{5 / 2}$ & 4.40071 & 4.38158 & 4.35784 & 4.34922 & 4.40695 & 4.41681 & $6.846-11$ \\
\hline 39 & $2 \mathrm{~s} 2 \mathrm{p} 3 \mathrm{~d}$ & ${ }^{4} \mathrm{~F}_{3 / 2}^{\circ}$ & 4.51231 & 4.45877 & 4.45181 & 4.44462 & 4.48920 & 4.52369 & 7.946-09 \\
\hline 40 & $2 \mathrm{~s} 2 \mathrm{p} 3 \mathrm{~d}$ & ${ }^{4} \mathrm{~F}_{5 / 2}^{\circ}$ & 4.51302 & 4.45946 & 4.45249 & 4.44530 & 4.49195 & 4.52369 & $6.564-09$ \\
\hline 41 & $2 \mathrm{~s} 2 \mathrm{p} 3 \mathrm{~d}$ & ${ }^{4} \mathrm{~F}_{7 / 2}^{\circ}$ & 4.51405 & 4.46049 & 4.45351 & 4.44629 & 4.49394 & 4.52461 & 6.321-09 \\
\hline 42 & $2 \mathrm{~s} 2 \mathrm{p} 3 \mathrm{~d}$ & ${ }^{4} \mathrm{~F}_{9 / 2}^{\circ}$ & 4.51545 & 4.46189 & 4.45491 & 4.44767 & 4.49306 & 4.52588 & $1.021-08$ \\
\hline 43 & $2 \mathrm{~s} 2 \mathrm{p}\left({ }^{3} \mathrm{P}^{\circ}\right) 3 \mathrm{p}$ & ${ }^{2} \mathrm{~S}_{1 / 2}$ & 4.49155 & 4.46847 & 4.46991 & 4.44991 & 4.50425 & 4.52115 & $6.799-11$ \\
\hline 44 & $2 s^{2} 4 p$ & ${ }^{2} \mathrm{P}_{1 / 2}^{\circ}$ & 4.55629 & 4.66966 & 4.48532 & 4.47085 & 4.51352 & & $6.042-10$ \\
\hline 45 & $2 s^{2} 4 p$ & ${ }^{2} \mathrm{P}_{3 / 2}^{\circ}$ & 4.55668 & 4.66919 & 4.48168 & 4.47122 & 4.51389 & 4.56407 & $6.091-10$ \\
\hline 46 & $2 \mathrm{~s} 2 \mathrm{p} 3 \mathrm{~d}$ & ${ }^{4} \mathrm{D}_{1 / 2}^{\circ}$ & 4.55421 & 4.50812 & 4.48131 & 4.47907 & 4.53457 & 4.56333 & $1.825-11$ \\
\hline 47 & $2 \mathrm{~s} 2 \mathrm{p} 3 \mathrm{~d}$ & ${ }^{4} \mathrm{D}_{3 / 2}^{\circ}$ & 4.55447 & 4.50836 & 4.48558 & 4.47933 & 4.53599 & 4.56358 & $1.826-11$ \\
\hline 48 & $2 \mathrm{~s} 2 \mathrm{p} 3 \mathrm{~d}$ & ${ }^{4} \mathrm{D}_{5 / 2}^{\mathrm{o}}$ & 4.55490 & 4.50876 & 4.48600 & 4.47975 & 4.53736 & 4.56397 & $1.828-11$ \\
\hline 49 & $2 \mathrm{~s} 2 \mathrm{p} 3 \mathrm{~d}$ & ${ }^{4} \mathrm{D}_{7 / 2}^{\circ}$ & 4.55549 & 4.50935 & 4.48659 & 4.48033 & 4.53663 & 4.56451 & $1.828-11$ \\
\hline 50 & $2 \mathrm{~s} 2 \mathrm{p}\left({ }^{3} \mathrm{P}^{\circ}\right) 3 \mathrm{~d}$ & ${ }^{2} \mathrm{D}_{3 / 2}^{\circ}$ & 4.57009 & 4.53461 & 4.51932 & 4.51168 & 4.56257 & 4.58533 & $4.520-11$ \\
\hline 51 & $2 \mathrm{~s} 2 \mathrm{p}\left({ }^{3} \mathrm{P}^{\circ}\right) 3 \mathrm{~d}$ & ${ }^{2} \mathrm{D}_{5 / 2}^{\circ / 2}$ & 4.57059 & 4.53591 & 4.51958 & 4.51200 & 4.56308 & 4.58579 & $4.371-11$ \\
\hline 52 & $2 \mathrm{~s} 2 \mathrm{p} 3 \mathrm{~d}$ & ${ }^{4} \mathrm{P}_{5 / 2}^{\circ / 2}$ & 4.59366 & 4.53415 & 4.52245 & 4.51564 & 4.56926 & 4.60176 & $3.387-11$ \\
\hline 53 & $2 \mathrm{~s} 2 \mathrm{p} 3 \mathrm{~d}$ & ${ }^{4} \mathrm{P}_{3 / 2}^{\circ}$ & 4.59469 & 4.53601 & 4.52326 & 4.51650 & 4.56990 & 4.60268 & $3.299-11$ \\
\hline 54 & $2 \mathrm{~s} 2 \mathrm{p} 3 \mathrm{~d}$ & ${ }^{4} \mathrm{P}_{1 / 2}^{\mathrm{o}}$ & 4.59536 & 4.53659 & 4.52390 & 4.51714 & 4.57139 & 4.60329 & $3.291-11$ \\
\hline 55 & $2 s^{2} 4 d$ & ${ }^{2} \mathrm{D}_{3 / 2}$ & 4.65265 & 4.76252 & 4.57274 & 4.56461 & 4.60070 & & $6.421-11$ \\
\hline 56 & $2 s^{2} 4 d$ & ${ }^{2} \mathrm{D}_{5 / 2}$ & 4.65269 & 4.76257 & 4.57279 & 4.56466 & 4.60075 & & $6.431-11$ \\
\hline 57 & $2 s^{2} 4 f$ & ${ }^{2} \mathrm{~F}_{5 / 2}^{\circ}$ & 4.67651 & 4.77532 & 4.59047 & 4.58285 & 4.62170 & & $1.673-10$ \\
\hline 58 & $2 s^{2} 4 f$ & ${ }^{2} \mathrm{~F}_{7 / 2}^{\circ}$ & 4.67661 & 4.77535 & 4.59051 & 4.58291 & 4.62171 & & $1.722-10$ \\
\hline 59 & $2 \mathrm{~s} 2 \mathrm{p}\left({ }^{3} \mathrm{P}^{\circ}\right) 3 \mathrm{~d}$ & ${ }^{2} \mathrm{~F}_{5 / 2}^{\circ}$ & 4.65425 & 4.62610 & 4.61403 & 4.60155 & 4.66080 & & $2.752-11$ \\
\hline 60 & $2 \mathrm{~s} 2 \mathrm{p}\left({ }^{3} \mathrm{P}^{\circ}\right) 3 \mathrm{~d}$ & ${ }^{2} \mathrm{~F}_{7 / 2}^{\circ}$ & 4.65637 & 4.62832 & 4.61622 & 4.60368 & 4.66288 & & $2.726-11$ \\
\hline 61 & $2 \mathrm{~s} 2 \mathrm{p}\left({ }^{3} \mathrm{P}^{\circ}\right) 3 \mathrm{~d}$ & ${ }^{2} \mathrm{P}_{3 / 2}^{\circ}$ & 4.68592 & 4.65671 & 4.64811 & 4.63243 & 4.68710 & & $2.752-11$ \\
\hline 62 & $2 \mathrm{~s} 2 \mathrm{p}\left({ }^{3} \mathrm{P}^{\circ}\right) 3 \mathrm{~d}$ & ${ }^{2} \mathrm{P}_{1 / 2}^{\circ}$ & 4.68730 & 4.65732 & 4.64954 & 4.63383 & 4.68842 & 4.56333 & $2.751-11$ \\
\hline
\end{tabular}


Table 1. b) Comparison of energy levels (in Ryd) of O IV and their lifetimes ( $\tau$ in s). $a \pm b \equiv a \times 10^{ \pm b}$.

\begin{tabular}{rlrrrrrrr}
\hline \hline Index & \multicolumn{1}{l}{ Configuration/Level } & NIST & GRASP1 & GRASP2 & GRASP3 & FAC & MCHF & $\tau$ \\
\hline 63 & $2 \mathrm{~s} 2 \mathrm{p}\left({ }^{1} \mathrm{P}^{\circ}\right) 3 \mathrm{~s}$ & ${ }^{2} \mathrm{P}_{1 / 2}^{\circ}$ & 4.72673 & 4.81499 & 4.75301 & 4.74747 & 4.76379 & $9.104-11$ \\
64 & $2 \mathrm{~s} 2 \mathrm{p}\left({ }^{1} \mathrm{P}^{\circ}\right) 3 \mathrm{~s}$ & ${ }^{2} \mathrm{P}_{3 / 2}^{\circ}$ & 4.72682 & 4.81517 & 4.75313 & 4.74758 & 4.76391 & $9.122-11$ \\
65 & $2 \mathrm{~s} 2 \mathrm{p}\left({ }^{1} \mathrm{P}^{\circ}\right) 3 \mathrm{p}$ & ${ }^{2} \mathrm{D}_{3 / 2}$ & 4.98760 & 5.08345 & 5.03332 & 5.01975 & 5.03978 & $2.135-10$ \\
66 & $2 \mathrm{~s} 2 \mathrm{p}\left({ }^{1} \mathrm{P}^{\circ}\right) 3 \mathrm{p}$ & ${ }^{2} \mathrm{D}_{5 / 2}$ & 4.98787 & 5.08377 & 5.03353 & 5.02008 & 5.03996 & $2.142-10$ \\
67 & $2 \mathrm{~s} 2 \mathrm{p}\left({ }^{1} \mathrm{P}^{\circ}\right) 3 \mathrm{p}$ & ${ }^{2} \mathrm{P}_{1 / 2}$ & 5.01007 & 5.10329 & 5.03586 & 5.03195 & 5.05500 & $1.016-10$ \\
68 & $2 \mathrm{~s} 2 \mathrm{p}\left({ }^{1} \mathrm{P}^{\circ}\right) 3 \mathrm{p}$ & ${ }^{2} \mathrm{P}_{3 / 2}$ & 5.01065 & 5.10382 & 5.03645 & 5.03254 & 5.05542 & $1.019-10$ \\
69 & $2 \mathrm{~s} 2 \mathrm{p}\left({ }^{1} \mathrm{P}^{\circ}\right) 3 \mathrm{p}$ & ${ }^{2} \mathrm{~S}_{1 / 2}$ & 5.05265 & 5.19023 & 5.11693 & 5.08386 & 5.10613 & $9.746-11$ \\
70 & $2 \mathrm{~s} 2 \mathrm{p}\left({ }^{1} \mathrm{P}^{\circ}\right) 3 \mathrm{~d}$ & ${ }^{2} \mathrm{~F}_{5 / 2}^{\circ}$ & 5.20143 & 5.33043 & 5.27749 & 5.26654 & 5.28775 & $3.402-11$ \\
71 & $2 \mathrm{~s} 2 \mathrm{p}\left({ }^{1} \mathrm{P}^{\circ}\right) 3 \mathrm{~d}$ & ${ }^{2} \mathrm{~F}_{7 / 2}^{\circ}$ & 5.20148 & 5.33027 & 5.27744 & 5.26674 & 5.28779 & $3.426-11$ \\
72 & $2 \mathrm{~s} 2 \mathrm{p}\left({ }^{1} \mathrm{P}^{\circ}\right) 3 \mathrm{~d}$ & ${ }^{2} \mathrm{D}_{3 / 2}^{\circ}$ & 5.24725 & 5.31967 & 5.30203 & 5.26732 & 5.29183 & $1.924-11$ \\
73 & $2 \mathrm{~s} 2 \mathrm{p}\left({ }^{1} \mathrm{P}^{\circ}\right) 3 \mathrm{~d}$ & ${ }^{2} \mathrm{D}_{5 / 2}^{\circ}$ & 5.24756 & 5.31989 & 5.30232 & 5.26778 & 5.29215 & $1.927-11$ \\
74 & $2 \mathrm{~s} 2 \mathrm{p}\left({ }^{1} \mathrm{P}^{\circ}\right) 3 \mathrm{~d}$ & ${ }^{2} \mathrm{P}_{1 / 2}^{\circ}$ & 5.30103 & 5.40584 & 5.35907 & 5.35312 & 5.37374 & $2.495-11$ \\
75 & $2 \mathrm{~s} 2 \mathrm{p}\left({ }^{1} \mathrm{P}^{\circ}\right) 3 \mathrm{~d}$ & ${ }^{2} \mathrm{P}_{3 / 2}^{\circ}$ & 5.30123 & 5.40592 & 5.35929 & 5.35333 & 5.37384 & $2.497-11$ \\
\hline
\end{tabular}

NIST: http://physics.nist.gov/PhysRefData.

GRASP1: energies from the GRASP code from 75 level calculations. GRASP2: energies from the GRASP code from 219 level calculations. GRASP3: energies from the GRASP code from 326 level calculations. FAC: energies from the FAC code with 686 level calculations.

MCHF: energies of Tayal (2006) from the MCHF code.

CIV3 calculations of Corrégé \& Hibbert (2002, 2004), which we discuss below.

In Table 1c we compare our energy levels from GRASP, corresponding to the GRASP2 calculations described above, with the experimental energies of NIST and theoretical results of Tachiev \& Froese-Fischer (2000), Tayal (2006), and Corrégé \& Hibbert (2004). The two MCHF calculations by Tachiev \& Froese-Fischer and Tayal generally agree with each other, except for the level $2\left(2 \mathrm{~s}^{2} 2 \mathrm{p}^{2} \mathrm{P}_{3 / 2}^{\circ}\right)$ for which Tayal's calculated energy is lower. Similarly, the CIV3 results of Corrégé \& Hibbert agree within $1 \%$ with the experimental or the MCHF energies of Tachiev \& Froese-Fischer. Hence, comparatively speaking, our energy levels are not as accurate as by Tachiev \& Froese-Fischer or Corrégé \& Hibbert, especially for the lowest 15 levels, as they differ from the experimental compilations by up to $0.12 \mathrm{Ryd}$, or on average by 0.064 Ryd. This is primarily because we have adopted the option of EAL as stated in Sect. 1. The other option of AL (average level) yields results similar to those presented in Table 1, because the orthonormal orbitals adopted in the calculations give an overall representation of all the configurations. To improve the energy levels, perhaps a better correlation for the lower levels/configurations is required as performed by Corrégé \& Hibbert.

\section{Radiative rates}

The absorption oscillator strength $\left(f_{i j}\right)$ and radiative rate $A_{j i}$ (in $\mathrm{s}^{-1}$ ) for a transition $i \rightarrow j$ are related by the following expression

$f_{i j}=\frac{m c}{8 \pi^{2} \mathrm{e}^{2}} \lambda_{j i}^{2} \frac{\omega_{j}}{\omega_{i}} A_{j i}=1.49 \times 10^{-16} \lambda_{j i}^{2}\left(\omega_{j} / \omega_{i}\right) A_{j i}$

where $m$ and $e$ are the electron mass and charge, respectively, $c$ is the velocity of light, $\lambda_{j i}$ is the transition energy/wavelength in $\AA$, and $\omega_{i}$ and $\omega_{j}$ are the statistical weights of the lower $(i)$ and upper $(j)$ levels, respectively. Similarly, the oscillator strength $f_{i j}$ (dimensionless) and the line strength $S$ (in atomic unit,
$1 \mathrm{au}=6.460 \times 10^{-36} \mathrm{~cm}^{2} \mathrm{esu}^{2}$ ) are related by the following standard equations.

For the electric dipole (E1) transitions

$A_{j i}=\frac{2.0261 \times 10^{18}}{\omega_{j} \lambda_{j i}^{3}} S^{\mathrm{E} 1} \quad$ and $\quad f_{i j}=\frac{303.75}{\lambda_{j i} \omega_{i}} S^{\mathrm{E} 1}$

for the magnetic dipole (M1) transitions

$A_{j i}=\frac{2.6974 \times 10^{13}}{\omega_{j} \lambda_{j i}^{3}} S^{\mathrm{M} 1} \quad$ and $\quad f_{i j}=\frac{4.044 \times 10^{-3}}{\lambda_{j i} \omega_{i}} S^{\mathrm{M} 1}$,

for the electric quadrupole (E2) transitions

$A_{j i}=\frac{1.1199 \times 10^{18}}{\omega_{j} \lambda_{j i}^{5}} S^{\mathrm{E} 2} \quad$ and $\quad f_{i j}=\frac{167.89}{\lambda_{j i}^{3} \omega_{i}} S^{\mathrm{E} 2}$,

and for the magnetic quadrupole (M2) transitions

$A_{j i}=\frac{1.4910 \times 10^{13}}{\omega_{j} \lambda_{j i}^{5}} S^{\mathrm{M} 2} \quad$ and $\quad f_{i j}=\frac{2.236 \times 10^{-3}}{\lambda_{j i}^{3} \omega_{i}} S^{\mathrm{M} 2}$.

As for energy levels, we have also performed a series of calculations for the radiative rates (and other related parameters) with increasing amount of CI. Compared in Table 2 are our results of oscillator strengths, for transitions among the lowest 20 levels and for which the $f$-values are comparatively large, obtained from the GRASP1, GRASP2, GRASP3 and FAC calculations, described above in Sect. 2. This comparison of $f$-values will enable us to assess the effect of CI on their accuracy. Also included in this table are the corresponding results of Tachiev \& Froese-Fischer (2000) and Tayal (2006), which are obtained from the MCHF code, and of Corrégé \& Hibbert (2004), which are obtained from the cIV3 code. The recommended $f$-values by NIST, which are available on their website at http://physics.nist.gov/PhysRefData, are also included in Table 2 . However, we would like to mention here that the $f$-values of NIST are primarily derived from the $L S$ calculations and hence the accuracy range for the transitions listed on their website is between B and D. 
Table 1. c) Comparison of energy levels (in Ryd) of O IV.

\begin{tabular}{|c|c|c|c|c|c|c|c|}
\hline Index & Configuratio & $\overline{\text { Level }}$ & NIST & GRASP & MCHF1 & MCHF2 & CIV3 \\
\hline 1 & $2 s^{2} 2 p$ & ${ }^{2} \mathrm{P}_{1 / 2}^{\circ}$ & 0.00000 & 0.00000 & 0.00000 & 0.00000 & 0.00000 \\
\hline 2 & $2 s^{2} 2 p$ & ${ }^{2} \mathrm{P}_{3 / 2}^{\circ}$ & 0.00352 & 0.00339 & 0.00353 & 0.00337 & 0.00348 \\
\hline 3 & $2 \mathrm{~s} 2 \mathrm{p}^{2}$ & ${ }^{4} \mathrm{P}_{1 / 2}$ & 0.65101 & 0.61517 & 0.65176 & 0.65294 & 0.64722 \\
\hline 4 & $2 \mathrm{~s} 2 \mathrm{p}^{2}$ & ${ }^{4} \mathrm{P}_{3 / 2}$ & 0.65220 & 0.61631 & 0.65296 & 0.65405 & 0.64840 \\
\hline 5 & $2 s 2 p^{2}$ & ${ }^{4} \mathrm{P}_{5 / 2}$ & 0.65388 & 0.61786 & 0.65464 & 0.65590 & 0.65005 \\
\hline 6 & $2 s 2 p^{2}$ & ${ }^{2} \mathrm{D}_{5 / 2}$ & 1.15673 & 1.21039 & 1.15877 & 1.17890 & 1.16049 \\
\hline 7 & $2 \mathrm{~s} 2 \mathrm{p}^{2}$ & ${ }^{2} \mathrm{D}_{3 / 2}$ & 1.15686 & 1.21054 & 1.15889 & 1.17885 & 1.16062 \\
\hline 8 & $2 s 2 p^{2}$ & ${ }^{2} \mathrm{~S}_{1 / 2}$ & 1.49782 & 1.56178 & 1.50125 & 1.53836 & 1.50381 \\
\hline 9 & $2 s 2 p^{2}$ & ${ }^{2} \mathrm{P}_{1 / 2}$ & 1.64466 & 1.74479 & 1.64665 & 1.68077 & 1.65056 \\
\hline 10 & $2 \mathrm{~s} 2 \mathrm{p}^{2}$ & ${ }^{2} \mathrm{P}_{3 / 2}$ & 1.64688 & 1.74688 & 1.64888 & 1.68289 & 1.65275 \\
\hline 11 & $2 p^{3}$ & ${ }^{4} \mathrm{~S}_{3 / 2}^{\circ}$ & 2.10993 & 2.11720 & 2.11236 & 2.11881 & 2.10546 \\
\hline 12 & $2 \mathrm{p}^{3}$ & ${ }^{2} \mathrm{D}_{5 / 2}^{\circ}$ & 2.32515 & 2.42052 & 2.32836 & 2.35208 & 2.32803 \\
\hline 13 & $2 \mathrm{p}^{3}$ & ${ }^{2} \mathrm{D}_{3 / 2}^{\circ / 2}$ & 2.32542 & 2.42085 & 2.32862 & 2.35181 & 2.32830 \\
\hline 14 & $2 \mathrm{p}^{3}$ & ${ }^{2} \mathrm{P}_{1 / 2}^{\circ}$ & 2.63370 & 2.75447 & 2.63854 & 2.67691 & 2.63981 \\
\hline 15 & $2 \mathrm{p}^{3}$ & ${ }^{2} \mathrm{P}_{3 / 2}^{\circ}$ & 2.63378 & 2.75455 & 2.63862 & 2.67701 & 2.63999 \\
\hline 16 & $2 s^{2} 3 s$ & ${ }^{2} \mathrm{~S}_{1 / 2}$ & 3.25882 & 3.18911 & 3.25983 & 3.25903 & 3.25614 \\
\hline 17 & $2 s^{2} 3 p$ & ${ }^{2} \mathrm{P}_{1 / 2}^{\circ}$ & 3.55541 & 3.48478 & 3.55854 & 3.54775 & 3.55804 \\
\hline 18 & $2 s^{2} 3 p$ & ${ }^{2} \mathrm{P}_{3 / 2}^{\circ}$ & 3.55620 & 3.48557 & 3.55934 & 3.55647 & 3.55885 \\
\hline 19 & $2 \mathrm{~s}^{2} 3 \mathrm{~d}$ & ${ }^{2} \mathrm{D}_{3 / 2}$ & 3.82307 & 3.75956 & 3.82510 & 3.83720 & 3.82306 \\
\hline 20 & $2 s^{2} 3 d$ & ${ }^{2} \mathrm{D}_{5 / 2}$ & 3.82323 & 3.75970 & 3.82525 & 3.83706 & 3.82321 \\
\hline 21 & $2 \mathrm{~s} 2 \mathrm{p} 3 \mathrm{~s}$ & ${ }^{4} \mathrm{P}_{1 / 2}^{\circ}$ & 3.99909 & 3.93307 & 4.00089 & 4.00397 & \\
\hline 22 & $2 \mathrm{~s} 2 \mathrm{p} 3 \mathrm{~s}$ & ${ }^{4} \mathrm{P}_{3 / 2}^{\circ}$ & 4.00032 & 3.93430 & 4.00213 & 4.00509 & \\
\hline 23 & $2 \mathrm{~s} 2 \mathrm{p} 3 \mathrm{~s}$ & ${ }^{4} \mathrm{P}_{5 / 2}^{\circ}$ & 4.00257 & 3.93654 & 4.00438 & 4.00712 & \\
\hline 24 & $2 \mathrm{~s} 2 \mathrm{p}\left({ }^{3} \mathrm{P}^{\circ}\right) 3 \mathrm{~s}$ & ${ }^{2} \mathrm{P}_{1 / 2}^{\circ}$ & 4.12628 & 4.08535 & 4.13060 & 4.14020 & \\
\hline 25 & $2 \mathrm{~s} 2 \mathrm{p}\left({ }^{3} \mathrm{P}^{\circ}\right) 3 \mathrm{~s}$ & ${ }^{2} \mathrm{P}_{3 / 2}^{\circ}$ & 4.12869 & 4.08774 & 4.13302 & 4.14239 & \\
\hline
\end{tabular}

NIST: http://physics. nist.gov/PhysRefData.

GRASP: energies from the GRASP code from 219 level calculations.

MCHF1: energies of Tachiev \& Froese-Fischer (2000) from the MCHF code.

MCHF2: energies of Tayal (2006) from the MCHF code.

CIV3: energies of Corrégé \& Hibbert (2004) from the cIV3 code.

Our $f$-values from the GRASP1 and GRASP2 calculations generally agree within $10 \%$, except for those transitions with upper levels 17 and 18 , such as $6-17,7-18,8-17$ and $10-18$, for which the differences are up to four orders of magnitude. All these transitions are comparatively weak and large differences between two calculations, performed with differing amount of $\mathrm{CI}$, arise from the addition and/or the cancellation of mixing coefficients. Clearly, the CI included in the GRASP1 calculations is inadequate as was also found for the energy levels in Sect. 2. Our GRASP2 calculations include a larger CI with the external orbitals as well as the configurations, and hence yield comparatively more accurate results for all transitions, including the weaker ones. This is confirmed by a comparison with the results obtained from the GRASP3 calculations, which include even larger CI. For the transitions shown in Table 2 the agreement between the $f$-values from the GRASP 2 and GRASP 3 calculations is better than $10 \%$ for all transitions. Similarly, the corresponding results obtained from the FAC code, by including a larger CI with the higher excited configurations/levels, are similar to those obtained from the GRASP2 and GRASP3 calculations. However, the differences for two weaker transitions $(8-17$ and $8-18)$ are up to $50 \%$, and the $f$-values from FAC are lower. This is due to the cancellation effects mentioned above. Therefore, we may conclude that the inclusion of a larger CI than that considered in the GRASP2 calculations is of no appreciable advantage as far as the $f$-values are concerned. This conclusion is in agreement with that in Sect. 2 regarding the energy levels, and therefore in our subsequent table we include our results for $f$-values from the GRASP2 calculations alone.
The $f$-values of Corrégé \& Hibbert (2004) obtained from the CIV3 code, of Tachiev \& Froese-Fischer (2000) obtained from the MCHF code, and those recommended by NIST agree within $\sim 10 \%$ with our final results from GRASP2, but only for strong transitions. For some weaker transitions, such as $6-17$, 7-18 and 8-18, our $f$-values are higher than those of others by up to a factor of two. This may be a consequence of a restricted CI included in our calculations, as a result of which the energy values not being highly accurate, as discussed already. Inclusion of $\mathrm{CI}$ is important for the determination of energy levels and/or the radiative rates, but only to a certain extent as also discussed and demonstrated in our earlier work on Mg-like ions (Aggarwal et al. 2007). We will also like to mention here that weaker transitions are more sensitive (and hence susceptible to change) with the inclusion/exclusion of CI. As a result of this their accuracy is always doubtful as also recently discussed in detail by Hibbert (2005). The other $f$-values listed in Table 2 are those of Tayal (2006), which have been obtained from the MCHF code. Again, for the strong transitions his $f$-values agree within $\sim 10 \%$ with ours as well as the other calculations. However, for some weaker transitions, particularly 8-17 and $10-18$, Tayal's $f$-values are lower by up to two orders of magnitude. This may be a consequence of the limited CI included by Tayal in the generation of his wavefunctions. Furthermore, we would like to point out two anomalies observed in Table 2 of Tayal. Firstly, his $f$ - and $A$-values are listed twice for the $2 \mathrm{~s} 2 \mathrm{p}^{2}{ }^{4} \mathrm{P}_{J}-2 \mathrm{~s} 2 \mathrm{p}\left({ }^{3} \mathrm{P}^{\circ}\right) 3 \mathrm{~d}{ }^{4} \mathrm{D}_{J}^{\circ}, 2 \mathrm{~s} 2 \mathrm{p}^{2}{ }^{4} \mathrm{P}_{J}-2 \mathrm{~s} 2 \mathrm{p}\left({ }^{3} \mathrm{P}^{\circ}\right) 3 \mathrm{~d}{ }^{4} \mathrm{P}_{J}^{\circ}$, and $2 \mathrm{~s} 2 \mathrm{p}^{2}{ }^{2} \mathrm{D}_{J}-2 \mathrm{~s} 2 \mathrm{p}\left({ }^{3} \mathrm{P}^{\circ}\right) 3 \mathrm{~s}{ }^{2} \mathrm{P}_{J}^{\circ}$ transitions. Secondly, and more importantly, some of the transitions involve the 
Table 2. Comparison of oscillator strengths ( $f$-values) for some transitions of O IV. $a-b \equiv a \times 10^{-b}$.

\begin{tabular}{|c|c|c|c|c|c|c|c|c|c|}
\hline I & $\overline{\bar{J}}$ & 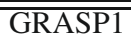 & 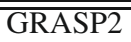 & " GRASP3 & $\overline{\overline{\text { FAC }}}$ & $\overline{\mathrm{MCHF} 1}$ & MCHF2 & $\overline{\text { CIV3 }}$ & $\overline{\text { NIST }}$ \\
\hline 1 & 6 & $1.210-1$ & $1.196-1$ & $1.215-1$ & $1.253-1$ & $1.117-1$ & $1.12-1$ & $1.112-1$ & $1.11-1$ \\
\hline 1 & 8 & $7.718-2$ & $8.030-2$ & $7.828-2$ & $7.652-2$ & $6.935-2$ & $7.06-2$ & $6.940-2$ & $6.71-2$ \\
\hline 1 & 9 & $2.363-1$ & $2.270-1$ & 2.281-1 & $2.318-1$ & $2.189-1$ & $2.28-1$ & $2.186-1$ & $2.24-1$ \\
\hline 1 & 10 & $1.187-1$ & $1.144-1$ & $1.149-1$ & $1.167-1$ & $1.105-1$ & $1.15-1$ & $1.103-1$ & $1.12-1$ \\
\hline 1 & 16 & $3.708-2$ & $3.350-2$ & $3.474-2$ & $3.294-2$ & $3.146-2$ & $2.84-2$ & $3.130-2$ & $3.14-2$ \\
\hline 1 & 19 & $5.177-1$ & $5.118-1$ & $5.147-1$ & $4.931-1$ & $5.077-1$ & $4.94-1$ & $4.952-1$ & $5.04-1$ \\
\hline 2 & 6 & $1.177-2$ & $1.161-2$ & $1.179-2$ & $1.217-2$ & $1.078-2$ & $1.09-2$ & $1.080-2$ & $1.10-2$ \\
\hline 2 & 7 & $1.080-1$ & $1.066-1$ & $1.083-1$ & $1.117-1$ & $9.954-2$ & $1.00-1$ & $9.910-2$ & $9.94-2$ \\
\hline 2 & 8 & $7.403-2$ & $7.601-2$ & 7.413-2 & $7.259-2$ & $6.462-2$ & $6.59-2$ & $6.480-2$ & $6.69-2$ \\
\hline 2 & 9 & $6.063-2$ & $5.887-2$ & $5.907-2$ & $5.991-2$ & $5.710-2$ & $5.95-2$ & $5.690-2$ & $5.57-2$ \\
\hline 2 & 10 & $2.983-1$ & $2.876-1$ & $2.889-1$ & 2.934-1 & $2.779-1$ & $2.90-1$ & $2.775-1$ & $2.79-1$ \\
\hline 2 & 16 & $3.720-2$ & $3.364-2$ & $3.487-2$ & 3.309-2 & $3.159-2$ & $2.85-2$ & $3.140-2$ & $3.14-2$ \\
\hline 2 & 19 & $5.178-2$ & $5.124-2$ & $5.153-2$ & $4.937-2$ & $5.013-2$ & $4.95-2$ & $4.960-2$ & $5.03-2$ \\
\hline 2 & 20 & $4.657-1$ & $4.608-1$ & 4.634-1 & $4.439-1$ & $4.508-1$ & $4.45-1$ & $4.460-1$ & $4.53-1$ \\
\hline 3 & 11 & $1.346-1$ & $1.303-1$ & $1.288-1$ & $1.321-1$ & $1.237-1$ & $1.27-1$ & $1.239-1$ & $1.25-1$ \\
\hline 4 & 11 & $1.344-1$ & $1.302-1$ & $1.287-1$ & $1.320-1$ & $1.236-1$ & $1.27-1$ & $1.238-1$ & $1.25-1$ \\
\hline 5 & 11 & $1.342-1$ & $1.300-1$ & $1.285-1$ & $1.318-1$ & $1.234-1$ & $1.27-1$ & $1.236-1$ & $1.25-1$ \\
\hline 6 & 12 & $1.302-1$ & $1.280-1$ & $1.241-1$ & $1.266-1$ & $1.178-1$ & $1.23-1$ & $1.181-1$ & $1.19-1$ \\
\hline 6 & 13 & $1.486-2$ & $1.467-2$ & $1.421-2$ & $1.450-2$ & $1.352-2$ & $1.32-2$ & $1.360-2$ & $1.33-2$ \\
\hline 6 & 14 & $1.013-1$ & $9.253-2$ & $9.274-2$ & $9.293-2$ & $8.174-2$ & $8.38-2$ & $8.190-2$ & $8.25-2$ \\
\hline 6 & 15 & $2.060-2$ & $1.885-2$ & $1.887-2$ & $1.888-2$ & $1.672-2$ & $1.64-2$ & $1.680-2$ & $1.65-2$ \\
\hline 6 & 17 & $1.574-2$ & $9.862-3$ & $1.006-2$ & $1.017-2$ & $5.330-3$ & $5.60-3$ & $5.300-3$ & $5.31-3$ \\
\hline 6 & 18 & $3.137-3$ & $1.967-3$ & $2.007-3$ & $2.032-3$ & $1.063-3$ & $1.13-3$ & $1.000-3$ & $1.06-3$ \\
\hline 7 & 12 & $1.012-2$ & $9.968-3$ & $9.655-3$ & $9.833-3$ & $9.222-3$ & $8.61-3$ & $9.300-3$ & $8.87-3$ \\
\hline 7 & 13 & $1.360-1$ & $1.338-1$ & $1.296-1$ & $1.321-1$ & $1.231-1$ & $1.26-1$ & $1.235-1$ & $1.24-1$ \\
\hline 7 & 15 & $1.205-1$ & $1.100-1$ & $1.103-1$ & $1.106-1$ & $9.706-2$ & $1.02-1$ & $9.730-2$ & $9.89-2$ \\
\hline 7 & 18 & $1.888-2$ & $1.181-2$ & $1.205-2$ & $1.220-2$ & 6.387-3 & $6.73-3$ & $6.300-3$ & $6.37-3$ \\
\hline 8 & 14 & $3.460-2$ & $3.724-2$ & $3.586-2$ & $3.818-2$ & $3.717-2$ & $4.22-2$ & $3.730-2$ & $3.91-2$ \\
\hline 8 & 15 & $7.297-2$ & $7.958-2$ & $7.656-2$ & $8.113-2$ & $7.999-2$ & $7.86-2$ & $8.010-2$ & $7.81-2$ \\
\hline 8 & 17 & $1.473-6$ & $4.986-3$ & $4.766-3$ & $2.901-3$ & & $1.88-5$ & $3.000-3$ & $3.15-3$ \\
\hline 8 & 18 & $1.299-6$ & $1.003-2$ & $9.588-3$ & $5.816-3$ & & $8.22-3$ & $6.100-3$ & $6.29-3$ \\
\hline 9 & 12 & $1.151-1$ & $1.318-1$ & $1.255-1$ & $1.269-1$ & $1.175-1$ & $1.13-1$ & $1.178-1$ & $1.17-1$ \\
\hline 9 & 14 & $1.348-1$ & $1.305-1$ & $1.276-1$ & $1.280-1$ & $1.133-1$ & $1.11-1$ & $1.137-1$ & $1.12-1$ \\
\hline 9 & 15 & $6.445-2$ & $6.152-2$ & $6.027-2$ & $6.053-2$ & $5.263-2$ & $5.95-2$ & $5.290-2$ & $5.62-2$ \\
\hline 9 & 17 & 6.764-4 & $1.497-4$ & $1.577-4$ & $1.202-4$ & & $1.88-5$ & $1.000-4$ & \\
\hline 10 & 12 & $1.105-2$ & $1.265-2$ & $1.206-2$ & $1.223-2$ & $1.122-2$ & $1.17-2$ & $1.130-2$ & $1.16-2$ \\
\hline 10 & 13 & $1.022-1$ & $1.170-1$ & $1.115-1$ & $1.128-1$ & $1.014-1$ & $1.02-1$ & $1.045-1$ & $1.04-1$ \\
\hline 10 & 14 & $3.315-2$ & $3.196-2$ & $3.127-2$ & $3.141-2$ & $2.760-2$ & $2.83-2$ & $2.770-2$ & $2.81-2$ \\
\hline 10 & 15 & $1.679-1$ & $1.621-1$ & $1.586-1$ & $1.591-1$ & $1.403-1$ & $1.39-1$ & $1.408-1$ & $1.41-1$ \\
\hline 10 & 18 & $8.473-4$ & $1.660-4$ & $1.765-4$ & $1.422-4$ & & $3.43-5$ & $1.000-4$ & \\
\hline 16 & 17 & $1.739-1$ & $1.910-1$ & $1.922-1$ & $1.903-1$ & $1.822-1$ & & $1.785-1$ & $1.83-1$ \\
\hline 16 & 18 & $3.488-1$ & $3.831-1$ & $3.856-1$ & $3.815-1$ & $3.652-1$ & & $3.580-1$ & $3.66-1$ \\
\hline 17 & 19 & $2.897-1$ & $3.128-1$ & $3.104-1$ & $3.075-1$ & 2.917-1 & & 2.876-1 & $2.96-1$ \\
\hline 18 & 19 & $2.892-2$ & $3.121-2$ & $3.097-2$ & $3.069-2$ & $2.910-2$ & & $2.870-2$ & $2.95-2$ \\
\hline 18 & 20 & $2.606-1$ & $2.811-1$ & $2.790-1$ & $2.764-1$ & $2.621-1$ & & $2.584-1$ & $2.67-1$ \\
\hline
\end{tabular}

GRASP1: present calculations from the GRASP code for 75 levels.

GRASP2: present calculations from the GRASP code for 219 levels.

GRASP3: present calculations from the GRASP code for 326 levels.

FAC: present calculations from the FAC code for 686 levels.

MCHF1: calculations of Tachiev \& Froese-Fischer (2000) from the MCHF code.

MCHF2: calculations of Tayal (2006) from the MCHF code.

CIV3: calculations of Corrégé \& Hibbert (2004) from the CIV3 code.

NIST: recommended values of NIST at http://physics.nist.gov/PhysRefData.

$2 \mathrm{~s} 2 \mathrm{p}\left({ }^{1} \mathrm{P}^{\circ}\right) 3 \mathrm{~s}{ }^{2} \mathrm{P}_{J}^{\circ}$ levels, for which he has not performed calculations as seen in his Table 1 . However, as stated earlier in Sect. 1, Tayal's $f$-values are available only for a limited number of transitions, and hence are inadequate for applications in plasma modelling.

In Table 3 we present transition energies/wavelengths $(\lambda$ in $\AA$ ), radiative rates $\left(A_{j i}\right.$ in $\left.\mathrm{s}^{-1}\right)$, oscillator strengths $\left(f_{i j}\right.$, dimensionless), and line strengths ( $S$, in au), in length form only, for all 1005 electric dipole (E1) transitions among the 75 levels of $\mathrm{O}$ IV. The indices used to represent the lower and upper levels of a transition have already been defined in Table 1a.
Similarly, there are 1196 electric quadrupole (E2), 1006 magnetic dipole (M1), and 1166 magnetic quadrupole (M2) transitions among the 75 levels. However, for these transitions only the $A$-values are listed in Table 3 , and the corresponding results for $f$ - or $S$-values can be easily obtained by using Eqs. (1)-(5).

A comparison of our $f$-values for some of the E1 transitions, and their accuracy assessment, was made in Table 2. However, those transitions are limited to the lowest 20 levels alone. Therefore, we now discuss the accuracy assessment for a larger number of transitions. One of the ways to assess the accuracy of the $f$ - or $A$-values is to compare the length and 
Table 4. Comparison of lifetimes $(\tau)$ for the lowest 25 levels of O IV.

\begin{tabular}{|c|c|c|c|c|c|c|}
\hline Index & Configuratio & $\overline{\text { Level }}$ & Present results & MCHF & Other theoretical & Experimental \\
\hline 1 & $2 s^{2} 2 p$ & ${ }^{2} \mathrm{P}_{1 / 2}^{\circ}$ & $\ldots$. & $\ldots$ & & \\
\hline 2 & $2 s^{2} 2 p$ & ${ }^{2} \mathrm{P}_{3 / 2}^{\circ}$ & $2.1675 \mathrm{ks}$ & $1.9160 \mathrm{a}$ & & \\
\hline 3 & $2 \mathrm{~s} 2 \mathrm{p}^{2}$ & ${ }^{4} \mathrm{P}_{1 / 2}^{3 / 2}$ & $0.5125 \mathrm{~ms}$ & $0.3380 \mathrm{a}$ & & \\
\hline 4 & $2 \mathrm{~s} 2 \mathrm{p}^{2}$ & ${ }^{4} \mathrm{P}_{3 / 2}$ & $5.9853 \mathrm{~ms}$ & $2.9990 \mathrm{a}$ & & \\
\hline 5 & $2 s 2 p^{2}$ & ${ }^{4} \mathrm{P}_{5 / 2}$ & $1.5281 \mathrm{~ms}$ & $0.8377 \mathrm{a}$ & & \\
\hline 6 & $2 \mathrm{~s} 2 \mathrm{p}^{2}$ & ${ }^{2} \mathrm{D}_{5 / 2}$ & $1.2080 \mathrm{~ns}$ & $1.4060 \mathrm{a}$ & $1.49 \mathrm{~b}$ & $1.64 \pm 0.008 \mathrm{~d}$ \\
\hline 7 & $2 s 2 p^{2}$ & ${ }^{2} \mathrm{D}_{3 / 2}$ & $1.1970 \mathrm{~ns}$ & $1.3960 \mathrm{a}$ & & $1.64 \pm 0.008 \mathrm{~d}$ \\
\hline 8 & $2 \mathrm{~s} 2 \mathrm{p}^{2}$ & ${ }^{2} \mathrm{~S}_{1 / 2}$ & $0.2200 \mathrm{~ns}$ & $0.2790 \mathrm{a}$ & $0.303 b, 0.249 c$ & $0.29 \pm 0.02 \mathrm{~d}, 0.36(8)$ \\
\hline 9 & $2 s 2 p^{2}$ & ${ }^{2} \mathrm{P}_{1 / 2}$ & $0.1206 \mathrm{~ns}$ & $0.1380 \mathrm{a}$ & $0.167 \mathrm{~b}$ & $0.158 \pm 0.007 \mathrm{~d}$ \\
\hline 10 & $2 \mathrm{~s} 2 \mathrm{p}^{2}$ & ${ }^{2} \mathrm{P}_{3 / 2}$ & $0.1205 \mathrm{~ns}$ & $0.1379 \mathrm{a}$ & & $0.158 \pm 0.007 \mathrm{~d}$ \\
\hline 11 & $2 p^{3}$ & ${ }^{4} S_{3 / 2}^{\circ}$ & $0.1425 \mathrm{~ns}$ & $0.1579 \mathrm{a}$ & $0.168 \mathrm{~b}$ & $0.198 \pm 0.008 \mathrm{~d}$ \\
\hline 12 & $2 \mathrm{p}^{3}$ & ${ }^{2} \mathrm{D}_{512}^{\circ}$ & $0.5076 \mathrm{~ns}$ & $0.5850 \mathrm{a}$ & $0.566 \mathrm{~b}$ & $0.63 \pm 0.02 \mathrm{~d}$ \\
\hline 13 & $2 \mathrm{p}^{3}$ & ${ }^{2} \mathrm{D}_{3 / 2}^{\mathrm{o} / 2}$ & $0.5082 \mathrm{~ns}$ & $0.5857 \mathrm{a}$ & & $0.58 \pm 0.02 \mathrm{~d}$ \\
\hline 14 & $2 \mathrm{p}^{3}$ & ${ }^{2} \mathrm{P}_{1 / 2}^{\mathrm{o} / 2}$ & $0.1805 \mathrm{~ns}$ & $0.2179 \mathrm{a}$ & $0.229 b$ & $0.246 \pm 0.009 d$ \\
\hline 15 & $2 \mathrm{p}^{3}$ & ${ }^{2} \mathrm{P}_{3 / 2}^{\circ}$ & $0.1808 \mathrm{~ns}$ & $0.2182 \mathrm{a}$ & & $0.227 \pm 0.011 \mathrm{~d}$ \\
\hline 16 & $2 s^{2} 3 s$ & ${ }^{2} S_{1 / 2}$ & $0.1218 \mathrm{~ns}$ & $0.1240 \mathrm{a}$ & $0.131 \mathrm{c}$ & $0.137(1) \mathrm{e}$ \\
\hline 17 & $2 s^{2} 3 p$ & ${ }^{2} \mathrm{P}_{1 / 2}^{\circ}$ & $0.9034 \mathrm{~ns}$ & $1.3870 \mathrm{a}$ & & $1.41 \pm 0.05 \mathrm{e}$ \\
\hline 18 & $2 s^{2} 3 p$ & ${ }^{2} \mathrm{P}_{3 / 2}^{\circ}$ & $0.9030 \mathrm{~ns}$ & $1.3860 \mathrm{a}$ & & $1.5 \pm 0.2 \mathrm{e}$ \\
\hline 19 & $2 s^{2} 3 d$ & ${ }^{2} \mathrm{D}_{3 / 2}$ & $0.0286 \mathrm{~ns}$ & $0.0282 \mathrm{a}$ & & \\
\hline 20 & $2 s^{2} 3 d$ & ${ }^{2} \mathrm{D}_{5 / 2}$ & $0.0286 \mathrm{~ns}$ & $0.0283 \mathrm{a}$ & & \\
\hline 21 & $2 \mathrm{~s} 2 \mathrm{p} 3 \mathrm{~s}$ & ${ }^{4} \mathrm{P}_{1 / 2}^{\circ}$ & $0.0943 \mathrm{~ns}$ & $0.0984 \mathrm{a}$ & $0.0983 \mathrm{c}$ & $0.101(5) \mathrm{e}$ \\
\hline 22 & $2 \mathrm{~s} 2 \mathrm{p} 3 \mathrm{~s}$ & ${ }^{4} \mathrm{P}_{3 / 2}^{\circ}$ & $0.0942 \mathrm{~ns}$ & $0.0982 \mathrm{a}$ & $0.0983 \mathrm{c}$ & $0.101(5) \mathrm{e}$ \\
\hline 23 & $2 \mathrm{~s} 2 \mathrm{p} 3 \mathrm{~s}$ & ${ }^{4} \mathrm{P}_{5 / 2}^{\circ}$ & $0.0941 \mathrm{~ns}$ & $0.0979 \mathrm{a}$ & $0.0983 \mathrm{c}$ & $0.101(5) \mathrm{e}$ \\
\hline 24 & $2 \mathrm{~s} 2 \mathrm{p}\left({ }^{3} \mathrm{P}^{\circ}\right) 3 \mathrm{~s}$ & ${ }^{2} \mathrm{P}_{1 / 2}^{\mathrm{o} / 2}$ & $0.1127 \mathrm{~ns}$ & $0.1418 \mathrm{a}$ & $1.40 \mathrm{c}$ & \\
\hline 25 & $2 \mathrm{~s} 2 \mathrm{p}\left({ }^{3} \mathrm{P}^{\circ}\right) 3 \mathrm{~s}$ & ${ }^{2} \mathrm{P}_{3 / 2}^{\circ}$ & $0.1122 \mathrm{~ns}$ & $0.1411 \mathrm{a}$ & $1.40 \mathrm{c}$ & \\
\hline
\end{tabular}

a: Tachiev \& Froese-Fischer (2000).

b: Safronova et al. (1999).

c: Nahar (1998).

d: Pinnington et al. (1974).

e: Pinnington et al. (1978).

f: Ishi et al. (1985).

velocity forms. However, we would first like to emphasize that such comparisons are only desirable and are not a necessary test for accuracy, because different calculations with differing amount of CI may generate comparable results in the two forms, but entirely dissimilar in magnitude - see Aggarwal et al. (2007) for various examples. Furthermore, the length forms are generally considered to be more reliable than the velocity forms (Hibbert 1975b).

For the stronger E1 transitions $(f \geq 0.01)$ listed in Table 3, the ratio $R=f_{L} / f_{V}\left(=A_{L} / A_{V}\right)$ is $>20 \%$ for 177 transitions, but is $>50 \%$ for only $56(\sim 5 \%)$. However, for 9 transitions (12-30, 13-31, 14-42, 15-42, 26-51, 28-50, 29-47, 59-73, and 60-72) the two forms differ by about an order of magnitude. Among the weaker transitions $(f<0.01)$, differences between the two forms are sometimes several orders of magnitude, and examples of such transitions are: $15-30\left(f=6.5 \times 10^{-9}\right)$, $35-57\left(f=1.4 \times 10^{-6}\right)$ and $50-65\left(f=2.9 \times 10^{-5}\right)$. Most of these transitions are very weak, and hence sensitive to mixing coefficients, as already discussed above, but do not affect the overall accuracy of the calculations. Therefore based on this and the comparison already made in Table 2, it may be fair to conclude that the $f$-values for a majority of the strong transitions are probably accurate to better than $20 \%$.

For other transitions (E2, M1 and M2) there are only a few calculations. For example, some unpublished results of Tachiev \& Froese-Fischer (2000) are available on their website http://www.vuse.vanderbilt.edu/ cff/mchf_collection/ for $4 \mathrm{E} 2,3 \mathrm{M} 1$ and $5 \mathrm{M} 2$ transitions among the lowest 5 levels of O IV. Differences between their $A$-values and our calculations are up to $50 \%$, with our $A$-values being invariably lower. However, with this limited comparison it is difficult to assess the accuracy of the reported $A$-values, particularly when a majority of these are very weak $\left(f \leq 10^{-5}\right)$.

\section{Lifetimes}

The lifetime $\tau$ for a level $j$ is defined as follows:

$\tau_{j}=\frac{1}{\sum_{i} A_{j i}}$

Since this is a measurable parameter, it provides a check on the accuracy of the calculations. Therefore, in Table $1 \mathrm{~b}$ we have also listed our calculated lifetimes, which include the contributions from four types of transitions, i.e. E1, E2, M1 and M2.

In Table 4 we compare our calculated lifetimes for the lowest 25 levels of O IV with the available theoretical (Nahar 1998; Safronova et al. 1999; Tachiev \& Froese-Fischer 2000) and experimental (Pinnington et al. 1974, 1978; Ishi et al. 1985) results. Lifetimes for all the listed levels are available only from the MCHF calculations of Tachiev \& Froese-Fischer which generally agree within $\sim 20 \%$ with our results. However, differences for the $2 \mathrm{~s} 2 \mathrm{p}^{2}{ }^{4} \mathrm{P}_{1 / 2,3 / 2,5 / 2}$ and $2 \mathrm{~s}^{2} 3 \mathrm{p}{ }^{2} \mathrm{P}_{1 / 2,3 / 2}^{\circ}$ levels are up to a factor of two. We would like to emphasize here that for the levels listed in Table 4 only the E1 transitions dominate (except for $2 s^{2} 2 p^{2} \mathrm{P}_{3 / 2}^{\circ}$ ), although contributions from E2, M1 and 
M2 transitions have also been included. Therefore, the differences observed here in Table 4 are primarily due to the differences in the calculated $A$-values for the electric dipole transitions alone. All transitions with upper $2 \mathrm{~s} 2 \mathrm{p}^{2}{ }^{4} \mathrm{P}_{1 / 2,3 / 2,5 / 2}$ and $2 \mathrm{~s}^{2} 3 \mathrm{p}{ }^{2} \mathrm{P}_{1 / 2,3 / 2}^{\circ}$ levels are weak $(f<0.01)$, and the $A$-values for such weak transitions are comparatively less reliable as already discussed in Sect. 3. Therefore, differences of up to a factor of two for some of the levels are not surprising. Other theoretical lifetimes are available for 5 levels from the work of Safronova et al. for which differences are up to $50 \%$, especially for the $2 \mathrm{~s} 2 \mathrm{p}^{2}{ }^{2} \mathrm{~S}_{1 / 2}$ level. Similarly, there is no discrepancy between the lifetimes calculated by Nahar and other workers, but only for the lower levels. For the highest two levels, namely $2 \mathrm{~s} 2 \mathrm{p}\left({ }^{3} \mathrm{P}^{\circ}\right) 3 \mathrm{~s}^{2} \mathrm{P}_{1 / 2,3 / 2}^{\circ}$, Nahar's results are higher than ours or those of Tachiev \& Froese-Fischer by an order of magnitude. Also, although Nahar listed her calculated lifetimes for these two levels as being in agreement with the measurements of Pinnington et al. (1978), this is not the case. Pinnington et al. have measured the lifetimes for the $2 \mathrm{~s}^{2} 3 \mathrm{p}^{2} \mathrm{P}^{\circ}$ levels and $n o t$ for the $2 \mathrm{~s} 2 \mathrm{p}\left({ }^{3} \mathrm{P}^{\circ}\right) 3 \mathrm{~s}{ }^{2} \mathrm{P}^{\circ}$ levels, as shown in the Table 4 of Nahar. Furthermore, we have performed a series of calculations, as already stated in Sects. 2 and 3, and our $\tau$-values for the two levels are consistent. Therefore, considering this consistency as well as the good agreement between our calculations and those of Tachiev \& Froese-Fischer, we conclude that the theoretical lifetimes of Nahar for the $2 \mathrm{~s} 2 \mathrm{p} 3 \mathrm{~s}{ }^{2} \mathrm{P}_{1 / 2,3 / 2}^{\circ}$ levels are overestimated by an order of magnitude. For the other levels for which measurements of lifetimes are available, there is no discrepancy between theory and experiment, although differences are sometimes up to $50 \%$, particularly for the $2 \mathrm{~s} 2 \mathrm{p}^{2}{ }^{2} \mathrm{D}_{3 / 2,5 / 2}$ and $2 \mathrm{~s}^{2} 3 \mathrm{p}^{2} \mathrm{P}_{1 / 2,3 / 2}^{\circ}$ levels.

\section{Collision strengths}

For the computation of collision strengths $\Omega$, we have employed the Dirac atomic R-matrix code (DARC), which includes the relativistic effects in a systematic way, in both the target description and the scattering model. It is based on the $j j$ coupling scheme, and uses the Dirac-Coulomb Hamiltonian in the $R$-matrix approach. The $R$-matrix radius has been adopted to be 16.32 au, and 35 continuum orbitals have been included for each channel angular momentum for the expansion of the wavefunction. This allows us to compute $\Omega$ up to an energy of $25 \mathrm{Ryd}$, sufficient to calculate the excitation rates up to a temperature of $10^{6} \mathrm{~K}$. The maximum number of channels for a partial wave is 322 , and the corresponding size of the Hamiltonian matrix is 11386 . In order to obtain convergence of $\Omega$ for all transitions and at all energies, we have included all partial waves with angular momentum $J \leq 40$, although a larger number would have been preferable for the convergence of some allowed transitions, especially at higher energies. However, to account for the inclusion of higher neglected partial waves, we have included a top-up, based on the Coulomb-Bethe approximation for allowed transitions and geometric series for others.

In Table 5 we list our values of $\Omega$ for resonance transitions at energies above thresholds. The indices used to represent the lower and upper levels of a transition have already been defined in Table 1a. No comparisons can be made with our calculations because neither Blum \& Pradhan (1992) nor Tayal (2006) have reported results for collision strengths. Therefore, in order to make an accuracy assessment of the values of $\Omega$, we have performed another calculation using the FAC code of $\mathrm{Gu}$ (2003). This code is also fully relativistic, and is based on

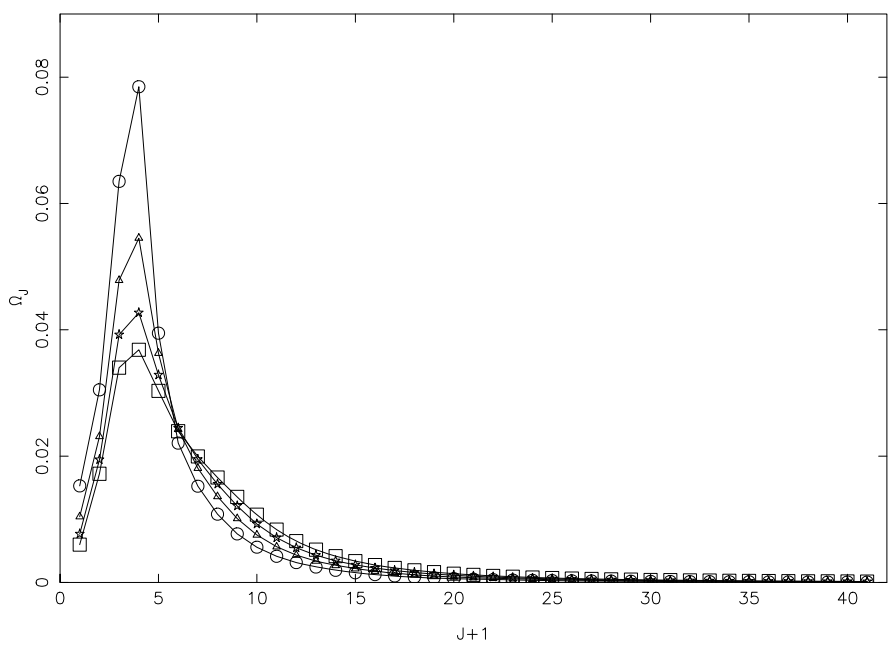

Fig. 1. Partial collision strengths for the $1-2\left(2 s^{2} 2 p^{2} P_{1 / 2}^{\circ}-2 s^{2} 2 p^{2} P_{3 / 2}^{\circ}\right)$ transition of $\mathrm{O}$ IV at 4 energies of 10 Ryd (circles), 15 Ryd (triangles), 20 Ryd (stars), and 25 Ryd (squares).

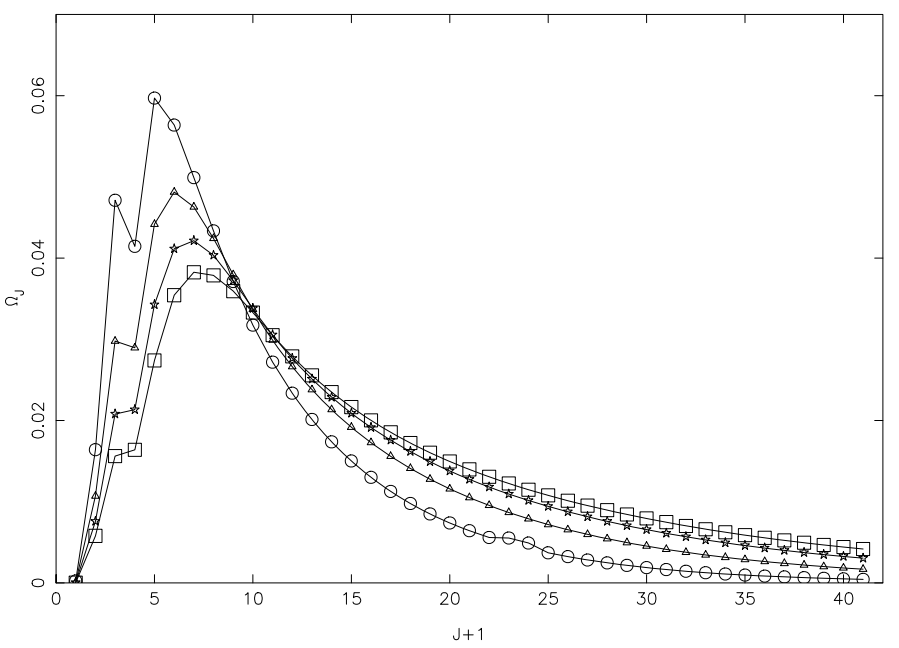

Fig. 2. Partial collision strengths for the $2-6\left(2 s^{2} 2 p^{2} P_{3 / 2}^{\circ}-2 s 2 p^{2}{ }^{2} D_{3 / 2}\right)$ transition of O IV at 4 energies of 10 Ryd (circles), 15 Ryd (triangles), 20 Ryd (stars), and 25 Ryd (squares).

the well known and widely used distorted-wave (DW) method. Furthermore, the same CI is included in FAC as in the calculations from DARC. Therefore, also included in Table 5 for a ready comparison are the $\Omega$ values from FAC at a single excited en$\operatorname{ergy}\left(E_{j}\right)$ of 25.9 Ryd. Generally the two sets of $\Omega$ agree well, but differences for some allowed transitions, such as 1-31, 1-32, 1-49 and 1-52, are striking. These differences are a direct consequence of the corresponding differences in the $f$-values.

In Figs. $1-3$ we show our variation of $\Omega$ with $J$ for three transitions, namely $1-2\left(2 \mathrm{~s}^{2} 2 \mathrm{p}^{2} \mathrm{P}_{1 / 2}^{\circ}-2 \mathrm{~s}^{2} 2 \mathrm{p}{ }^{2} \mathrm{P}_{3 / 2}^{\circ}\right), 2-6$ $\left(2 s^{2} 2 p^{2} \mathrm{P}_{3 / 2}^{\circ}-2 s 2 p^{2}{ }^{2} \mathrm{D}_{3 / 2}\right)$, and $19-20\left(2 \mathrm{~s}^{2} 3 \mathrm{~d}{ }^{2} \mathrm{D}_{3 / 2}-2 \mathrm{~s}^{2} 3 \mathrm{~d}\right.$ ${ }^{2} \mathrm{D}_{5 / 2}$ ), respectively. The $1-2$ and $19-20$ transitions are forbidden (within the odd and even parity configurations), and 2-6 is allowed. For the $1-2$ resonance transition values of $\Omega$ have converged within $J \leq 20$ at all energies as shown in Fig. 1, while for some forbidden transitions among excited levels, such as 19-20 shown in Fig. 3, the convergence of $\Omega$ is slower, but is completely within the $J \leq 40$ range. However, for the allowed 


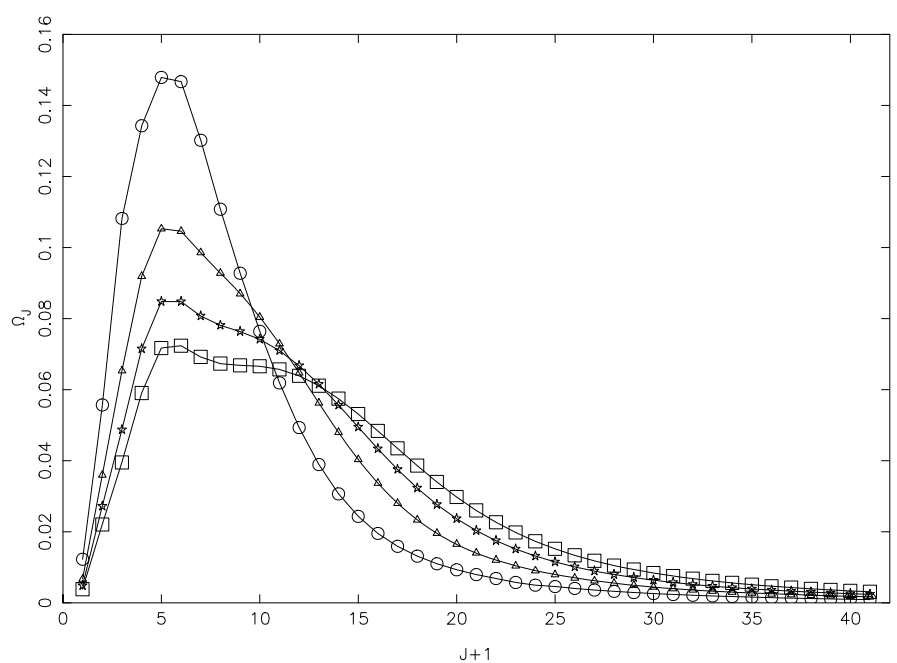

Fig. 3. Partial collision strengths for the $19-20\left(2 s^{2} 3 d{ }^{2} D_{3 / 2}-2 s^{2} 3 d\right.$ ${ }^{2} \mathrm{D}_{5 / 2}$ ) transition of O IV at 4 energies of 10 Ryd (circles), 15 Ryd (triangles), 20 Ryd (stars), and 25 Ryd (squares).

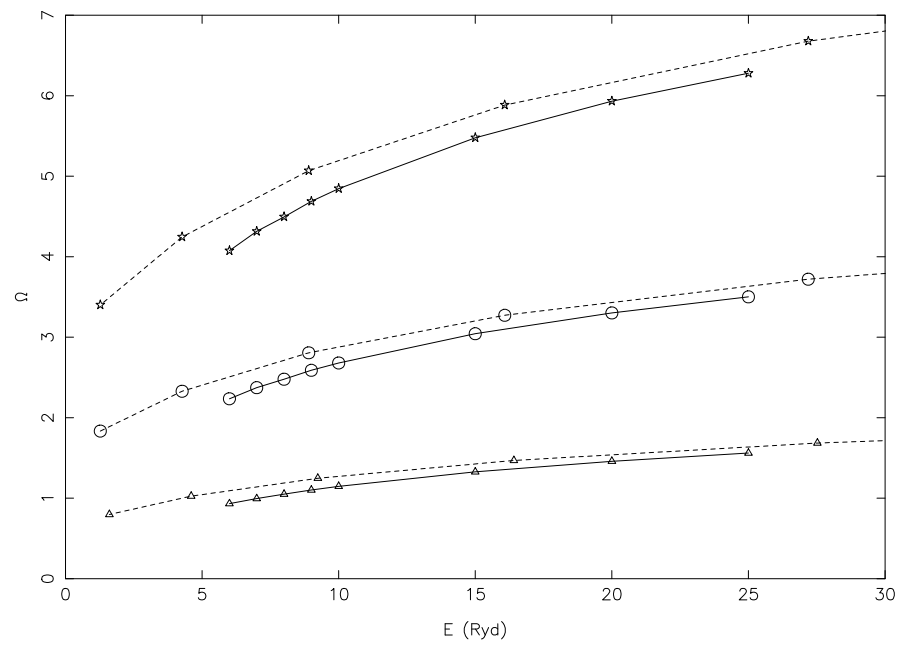

Fig. 4. Comparison of collision strengths from the DARC (continuous curves) and FAC (broken curves) codes for the $1-6\left(2 \mathrm{~s}^{2} 2 \mathrm{p}^{2} \mathrm{P}_{1 / 2}^{\circ}-2 \mathrm{~s} 2 \mathrm{p}^{2}\right.$ ${ }^{2} \mathrm{D}_{3 / 2}$ : circles), $1-8\left(2 \mathrm{~s}^{2} 2 \mathrm{p}^{2} \mathrm{P}_{1 / 2}^{\circ}-2 \mathrm{~s} 2 \mathrm{p}^{2}{ }^{2} \mathrm{~S}_{1 / 2}\right.$ : triangles $)$ and $2-7\left(2 \mathrm{~s}^{2} 2 \mathrm{p}\right.$ ${ }^{2} \mathrm{P}_{3 / 2}^{\circ}-2 \mathrm{~s} 2 \mathrm{p}^{2}{ }^{2} \mathrm{D}_{5 / 2}$ : stars) allowed transitions of O IV.

transitions our wide range of partial waves is just sufficient for the convergence of $\Omega$, as shown in Fig. 2. For such transitions a top-up has been included as mentioned above. In conclusion we may state that our range of $J$ values is fully sufficient for the convergence of $\Omega$ values for all transitions and at all energies.

In Fig. 4 we show the variation of our values of $\Omega$ with energy for three allowed transitions, namely $1-6\left(2 \mathrm{~s}^{2} 2 \mathrm{p}\right.$ $\left.{ }^{2} \mathrm{P}_{1 / 2}^{\circ}-2 \mathrm{~s} 2 \mathrm{p}^{2}{ }^{2} \mathrm{D}_{3 / 2}\right), 1-8\left(2 \mathrm{~s}^{2} 2 \mathrm{p}{ }^{2} \mathrm{P}_{1 / 2}^{\circ}-2 \mathrm{~s} 2 \mathrm{p}^{2}{ }^{2} \mathrm{~S}_{1 / 2}\right)$ and $2-7$ $\left(2 s^{2} 2 p^{2} \mathrm{P}_{3 / 2}^{\circ}-2 s 2 p^{2}{ }^{2} \mathrm{D}_{5 / 2}\right)$. Also included in this figure are the corresponding results obtained from the FAC code. For all the above three (and many other) transitions there are no discrepancies between the $f$-values obtained from the two independent (GRASP and FAC) codes, and therefore the $\Omega$ values also agree to better than $10 \%$. However, the $\Omega$ values obtained from FAC are slightly higher, particularly towards the lower end of the energy range, and the agreement between the two calculations improves with increasing energy. This slight difference in values of $\Omega$ is expected because the DW method generally

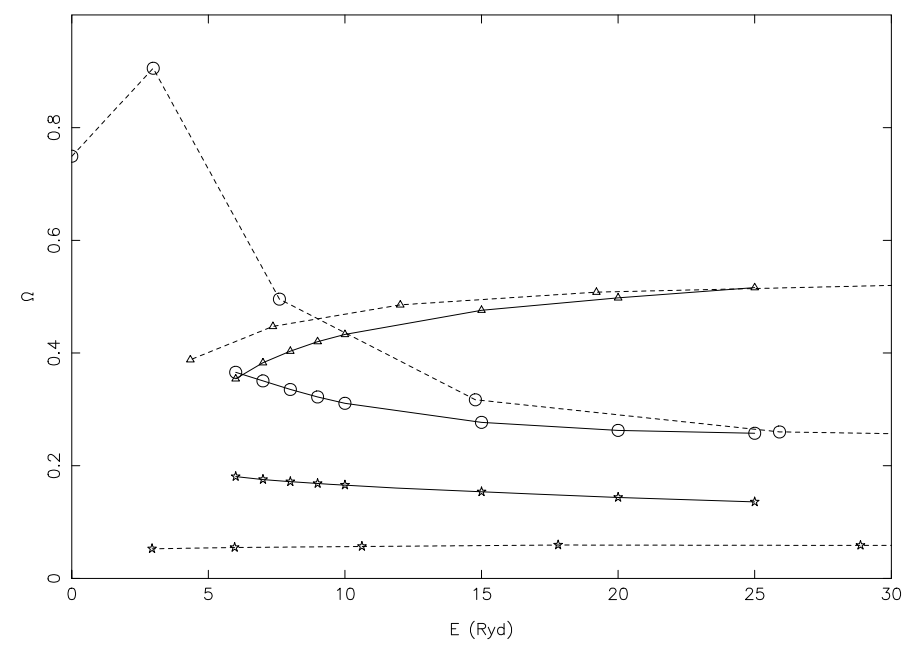

Fig. 5. Comparison of collision strengths from the DARC (continuous curves) and FAC (broken curves) codes for the 1-2 (2s $2 \mathrm{p}^{2} \mathrm{P}_{1 / 2}^{\circ}-2 \mathrm{~s}^{2} 2 \mathrm{p}$ ${ }^{2} \mathrm{P}_{3 / 2}^{\circ}$ : circles $), 1-24\left(2 \mathrm{~s}^{2} 2 \mathrm{p}{ }^{2} \mathrm{P}_{1 / 2}^{\circ}-2 \mathrm{~s} 2 \mathrm{p}\left({ }^{3} \mathrm{P}^{\circ}\right) 3 \mathrm{~s}{ }^{2} \mathrm{P}_{1 / 2}^{\circ}\right.$ : triangles $)$ and $2-15\left(2 \mathrm{~s}^{2} 2 \mathrm{p}^{2} \mathrm{P}_{3 / 2}^{\circ}-2 \mathrm{p}^{3}{ }^{2} \mathrm{P}_{3 / 2}^{\circ}\right.$ : stars $)$ forbidden transitions of $\mathrm{O}$ IV.

overestimates the results at lower energies due to the exclusion of channel coupling.

Similar comparisons between the two calculations are made in Fig. 5 for three forbidden transitions, namely $1-2\left(2 \mathrm{~s}^{2} 2 \mathrm{p}\right.$ $\left.{ }^{2} \mathrm{P}_{1 / 2}^{\circ}-2 \mathrm{~s}^{2} 2 \mathrm{p}^{2} \mathrm{P}_{3 / 2}^{\circ}\right), 1-24\left(2 \mathrm{~s}^{2} 2 \mathrm{p}^{2} \mathrm{P}_{1 / 2}^{\circ}-2 \mathrm{~s} 2 \mathrm{p}\left({ }^{3} \mathrm{P}^{\circ}\right) 3 \mathrm{~s}^{2} \mathrm{P}_{1 / 2}^{\circ}\right)$ and $2-15\left(2 \mathrm{~s}^{2} 2 \mathrm{p}^{2} \mathrm{P}_{3 / 2}^{\circ}-2 \mathrm{p}^{3}{ }^{2} \mathrm{P}_{3 / 2}^{\circ}\right)$. For these transitions also the agreement between the two calculations improves with increasing energy, although differences for the 2-15 (and some other) transition(s) remain in the entire energy range. Similarly, the $\Omega$ value from FAC at an energy of 3 Ryd for the 1-2 transition is anomalous. Such occasional anomalies for a few random transitions occur because of the interpolation and extrapolation techniques employed in the FAC code, which is designed to generate a large amount of atomic data in a comparatively very short period of time, and without too much loss of accuracy. Therefore, sometimes a problem of a few anomalies may arise from the calculations from FAC, but overall we observe no discrepancy with our calculations performed from the DARC code, as also observed for many other ions, such as of iron - see, for example, Aggarwal \& Keenan (2006) and the references therein.

Based on the discussion above and the comparisons made we do not see any deficiency in our calculations for $\Omega$. However, scope remains for improvement mainly because the wavefunctions adopted for calculating the values of $\Omega$ are from our GRASP1 calculations. Our GRASP2 and GRASP3 calculations did improve the accuracy of the wavefunctions, as already discussed in Sects. 2 and 3, yet these wavefunctions have not been employed in DARC, because the collisional calculations become lengthy due to the mixing of the levels. For example, a calculation for the desired 75 levels of Table 1 and adopting the wavefunctions from either GRASP2 or GRASP3 will require an inclusion of at least 120 levels. A calculation involving a larger number of levels may be possible in the future, which may improve the $\Omega$ values, and will particularly be useful for the determination of excitation rates (discussed in Sect. 6), because of the resonances.

Finally, we would like to state here that measurements of cross sections (and hence collision strengths) are available, in a limited energy range below $19.3 \mathrm{eV}$ (Smith et al. 2003), for the $2 s^{2} 2 p^{2} \mathrm{P}^{\circ}-2 \mathrm{~s} 2 \mathrm{p}^{2}{ }^{4} \mathrm{P}$ and $2 \mathrm{~s} 2 \mathrm{p}^{2}{ }^{2} \mathrm{D}$ transitions. In Figs. 6 and 7 


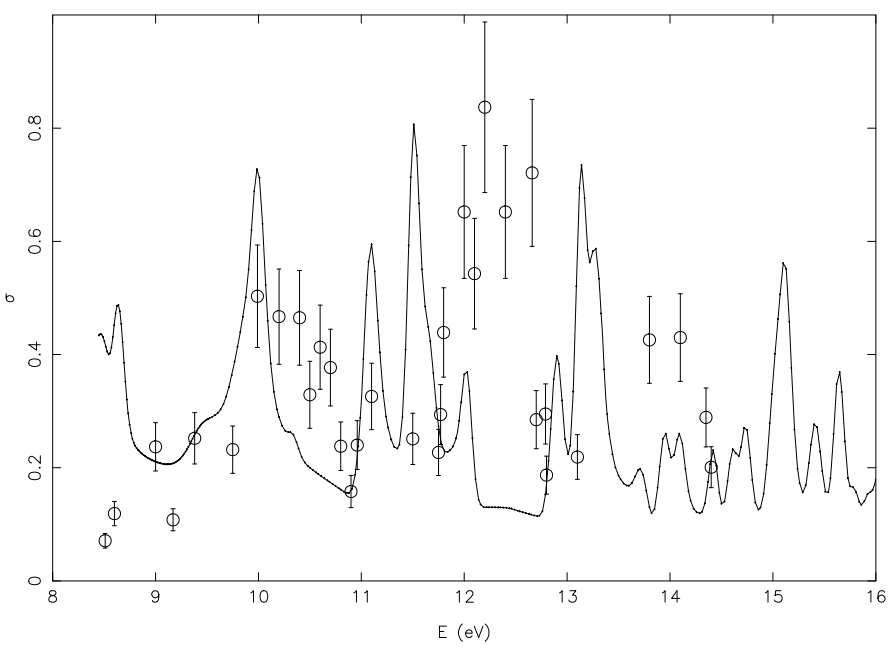

Fig. 6. Comparison of theoretical (continuous curve) and experimental (circles: Smith et al. 2003) cross sections (in units of $10^{-16} \mathrm{~cm}^{2}$ ) for the $2 \mathrm{~s}^{2} 2 \mathrm{p}^{2} \mathrm{P}^{\circ}-2 \mathrm{~s} 2 \mathrm{p}^{2}{ }^{4} \mathrm{P}$ transition of O IV.

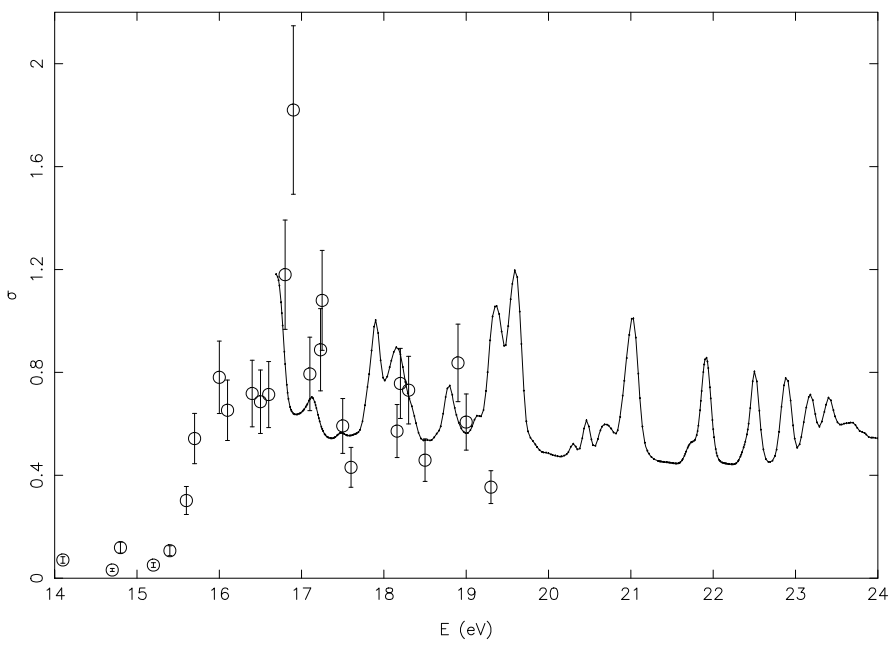

Fig. 7. Comparison of theoretical (continuous curve) and experimental (circles: Smith et al. 2003) cross sections (in units of $10^{-16} \mathrm{~cm}^{2}$ ) for the $2 \mathrm{~s}^{2} 2 \mathrm{p}^{2} \mathrm{P}^{\circ}-2 \mathrm{~s} 2 \mathrm{p}^{2}{ }^{2} \mathrm{D}$ transition of O IV.

we compare our cross sections $\left(\sigma\right.$, in units of $\left.10^{-16} \mathrm{~cm}^{2}\right)$ with the measurements of Smith et al. for the $2 s^{2} 2 p^{2} \mathrm{P}^{\circ}-2 \mathrm{~s} 2 \mathrm{p}^{2}{ }^{4} \mathrm{P}$ and $2 \mathrm{~s} 2 \mathrm{p}^{2}{ }^{2} \mathrm{D}$ transition, respectively. A similar comparison for both of these transitions was shown by Smith et al. and Tayal (2006) in their (his) Figs. 3 and 4. Similarly, as was done by them, we too have convoluted the cross sections data with the experimental width $\Delta E$ of $0.104 \mathrm{eV}$. For both transitions, and especially for $2 s^{2} 2 p^{2} \mathrm{P}^{\circ}-2 \mathrm{~s} 2 \mathrm{p}^{2}{ }^{4} \mathrm{P}$, agreement between theory and experiment was comparatively better with the calculations of Tayal. This is mainly because his energies for the levels under consideration are more accurate as shown in Table 1c and discussed in Sect. 2. Therefore, there is scope for improvement over our calculations, particularly at very low energies. Nevertheless, similar measurements for a few more transitions, and preferably for fine-structure transitions over a wider energy range, will be highly useful in further assessing the accuracy of the present calculations.

\section{Excitation rates}

Excitation rates, along with energy levels and radiative rates, are required for plasma modelling, and are determined from the collision strengths $(\Omega)$. Since the threshold energy region is dominated by numerous closed-channel (Feshbach) resonances, values of $\Omega$ need to be calculated in a fine energy mesh in order to accurately account for their contribution. Furthermore, in a hot plasma electrons have a wide distribution of velocities, and therefore values of $\Omega$ are generally averaged over a Maxwellian distribution as follows:

$\Upsilon\left(T_{\mathrm{e}}\right)=\int_{0}^{\infty} \Omega(E) \exp \left(-E_{j} / k T_{\mathrm{e}}\right) \mathrm{d}\left(E_{j} / k T_{\mathrm{e}}\right)$

where $k$ is Boltzmann constant, $T_{\mathrm{e}}$ is the electron temperature in $K$, and $E_{j}$ is the electron energy with respect to the final (excited) state. Once the value of $\Upsilon$ is known the corresponding results for the excitation $q(i, j)$ and de-excitation $q(j, i)$ rates can be easily obtained from the following equations:

$q(i, j)=\frac{8.63 \times 10^{-6}}{\omega_{i} T_{\mathrm{e}}^{1 / 2}} \Upsilon \exp \left(-E_{i j} / k T_{\mathrm{e}}\right) \mathrm{cm}^{3} \mathrm{~s}^{-1}$

and

$q(j, i)=\frac{8.63 \times 10^{-6}}{\omega_{j} T_{\mathrm{e}}^{1 / 2}} \Upsilon \mathrm{cm}^{3} \mathrm{~s}^{-1}$,

where $\omega_{i}$ and $\omega_{j}$ are the statistical weights of the initial $(i)$ and final $(j)$ states, respectively, and $E_{i j}$ is the transition energy. The contribution of resonances may enhance the values of $\Upsilon$ over those of the background values of collision strengths $\left(\Omega_{\mathrm{B}}\right)$, especially for the forbidden transitions, by up to a factor of ten (or even more) depending on the transition and/or the temperature. Similarly, values of $\Omega$ need to be calculated over a wide energy range (above thresholds) in order to obtain convergence of the integral in Eq. (7).

We have computed values of $\Omega$ at over 3500 energies in the threshold region with $\Delta E \leq 0.002$ Ryd. This fine energy mesh ensures to a large extent that neither a majority of resonances are missed, nor do the exceptionally high resonances have unreasonably large width. In Figs. 8-11 we show resonances for only four transitions, namely $1-2\left(2 \mathrm{~s}^{2} 2 \mathrm{p}^{2} \mathrm{P}_{1 / 2}^{\circ}-2 \mathrm{~s}^{2} 2 \mathrm{p}{ }^{2} \mathrm{P}_{3 / 2}^{\circ}\right), 1-3$ $\left(2 \mathrm{~s}^{2} 2 \mathrm{p}{ }^{2} \mathrm{P}_{1 / 2}^{\circ}-2 \mathrm{~s} 2 \mathrm{p}^{2}{ }^{4} \mathrm{P}_{1 / 2}\right), 1-6\left(2 \mathrm{~s}^{2} 2 \mathrm{p}^{2} \mathrm{P}_{1 / 2}^{\circ}-2 \mathrm{~s} 2 \mathrm{p}^{2}{ }^{2} \mathrm{D}_{3 / 2}\right)$, and 2-7 $\left(2 \mathrm{~s}^{2} 2 \mathrm{p}^{2} \mathrm{P}_{3 / 2}^{\circ}-2 \mathrm{~s} 2 \mathrm{p}^{2}{ }^{2} \mathrm{D}_{5 / 2}\right)$. These transitions have specifically been chosen because similar results from the calculations of Tayal (2006) are available for comparison. For all transitions the resonances in $\Omega$ shown in the present Figs. 8-11 are comparable with the corresponding results of Tayal in his Figs. 1-4. However, a minor correction is required in his caption of Fig. 3 as the transition is $1-6\left(2 \mathrm{~s}^{2} 2 \mathrm{p}^{2} \mathrm{P}_{1 / 2}^{\circ}-2 \mathrm{~s} 2 \mathrm{p}^{2}{ }^{2} \mathrm{D}_{3 / 2}\right)$ and not $2-6$ $\left(2 \mathrm{~s}^{2} 2 \mathrm{p}^{2} \mathrm{P}_{3 / 2}^{\circ}-2 \mathrm{~s} 2 \mathrm{p}^{2}{ }^{2} \mathrm{D}_{3 / 2}\right)$ as stated by him.

Our results for $\Upsilon$ for all transitions among the lowest 75 levels of $\mathrm{O}$ IV are listed in Table 6 over a wide temperature range up to $10^{6} \mathrm{~K}$, suitable for applications in a variety of plasmas. Earlier available results from the $R$-matrix code are by Blum \& Pradhan (1992) and Tayal (2006). Blum \& Pradhan reported values of $\Upsilon$ among the lowest 15 levels, but only up to $T_{\mathrm{e}}=4 \times$ $10^{4} \mathrm{~K}$, inadequate for applications to solar plasmas. Realising this, Zhang et al. (1994) extended the calculations of Blum \& Pradhan up to $T_{\mathrm{e}}=4.5 \times 10^{5} \mathrm{~K}$, but reported results only for transitions among the lowest 7 levels. Tayal therefore performed a larger calculation as mentioned in Sect. 1 . He included the lowest 54 levels and reported values of $\Upsilon$ up to $T_{\mathrm{e}}=4.0 \times 10^{5} \mathrm{~K}$. 


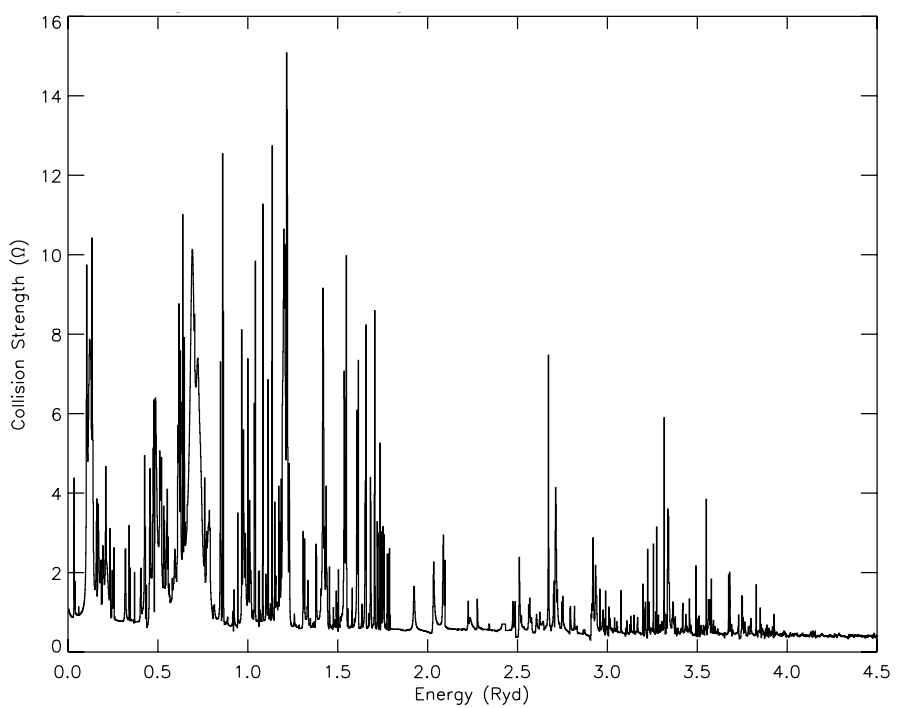

Fig. 8. Collision strengths for the $1-2\left(2 s^{2} 2 p^{2} \mathrm{P}_{1 / 2}^{\circ}-2 s^{2} 2 p^{2} \mathrm{P}_{3 / 2}^{\circ}\right)$ transition of O IV.

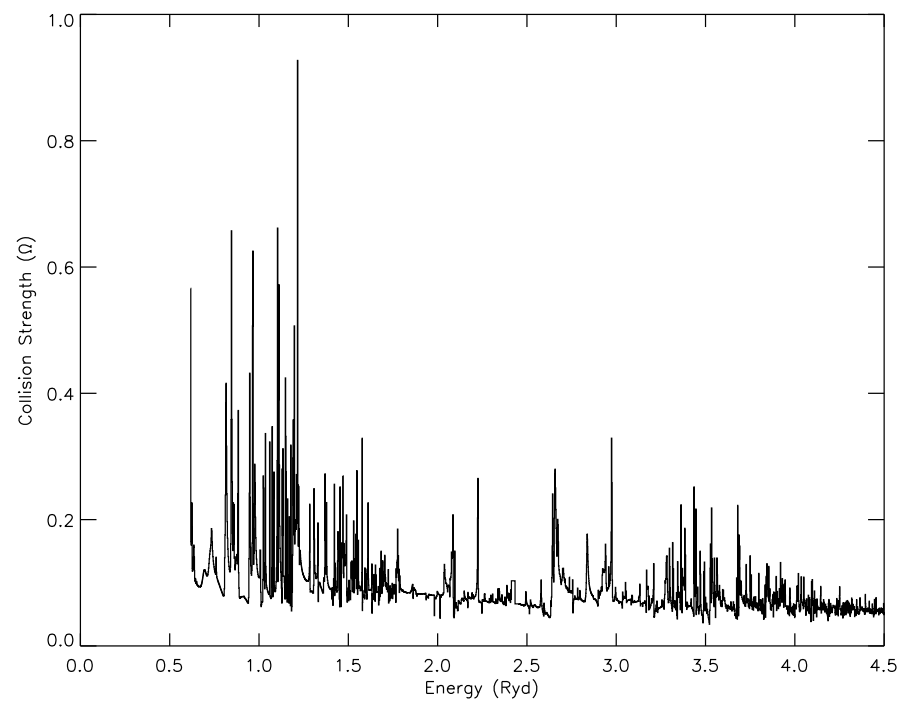

Fig. 9. Collision strengths for the $1-3\left(2 \mathrm{~s}^{2} 2 \mathrm{p}^{2} \mathrm{P}_{1 / 2}^{\circ}-2 \mathrm{~s} 2 \mathrm{p}^{2}{ }^{4} \mathrm{P}_{1 / 2}\right)$ transition of O IV.

Furthermore, he included a larger range of partial waves with $J \leq 25$ in order to achieve a better convergence of $\Omega$ up to an energy of 20 Ryd. For most of the (forbidden) transitions this range of partial waves is sufficient for the convergence of $\Omega$, but a higher range is preferable as already demonstrated in Figs. 2 and 3. Finally, he also included the one-body relativistic operators in a Breit-Pauli approximation in order to obtain values of $\Omega$ and $\Upsilon$ for fine-structure transitions. For a lighter ion such as $\mathrm{O}$ IV this approach is adequate, although we have preferred to employ the fully relativistic approach of the DARC code.

In Figs. 12-17 we compare our values of $\Upsilon$ with those of Blum \& Pradhan (1992), Zhang et al. (1994) and Tayal (2006) for six representative transitions, namely 1-2 $\left(2 \mathrm{~s}^{2} 2 \mathrm{p}^{2} \mathrm{P}_{1 / 2}^{\circ}-2 \mathrm{~s}^{2} 2 \mathrm{p}^{2} \mathrm{P}_{3 / 2}^{\circ}\right), 2-3\left(2 \mathrm{~s}^{2} 2 \mathrm{p}^{2} \mathrm{P}_{3 / 2}^{\circ}-2 \mathrm{~s} 2 \mathrm{p}^{2}{ }^{4} \mathrm{P}_{1 / 2}\right), 2-4$ $\left(2 \mathrm{~s}^{2} 2 \mathrm{p}^{2} \mathrm{P}_{3 / 2}^{\circ}-2 \mathrm{~s} 2 \mathrm{p}^{2}{ }^{4} \mathrm{P}_{3 / 2}\right), 2-5\left(2 \mathrm{~s}^{2} 2 \mathrm{p}^{2} \mathrm{P}_{3 / 2}^{\circ}-2 \mathrm{~s} 2 \mathrm{p}^{2}{ }^{4} \mathrm{P}_{5 / 2}\right), 2-6$ $\left(2 \mathrm{~s}^{2} 2 \mathrm{p}^{2} \mathrm{P}_{3 / 2}^{\circ}-2 \mathrm{~s} 2 \mathrm{p}^{2}{ }^{2} \mathrm{D}_{3 / 2}\right)$, and $2-7\left(2 \mathrm{~s}^{2} 2 \mathrm{p}^{2} \mathrm{P}_{3 / 2}^{\circ}-2 \mathrm{~s} 2 \mathrm{p}^{2}{ }^{2} \mathrm{D}_{5 / 2}\right)$. The results of Blum \& Pradhan and Zhang et al. should be the same in the common temperature range below $4 \times 10^{4} \mathrm{~K}$, because there is no difference between the two calculations.

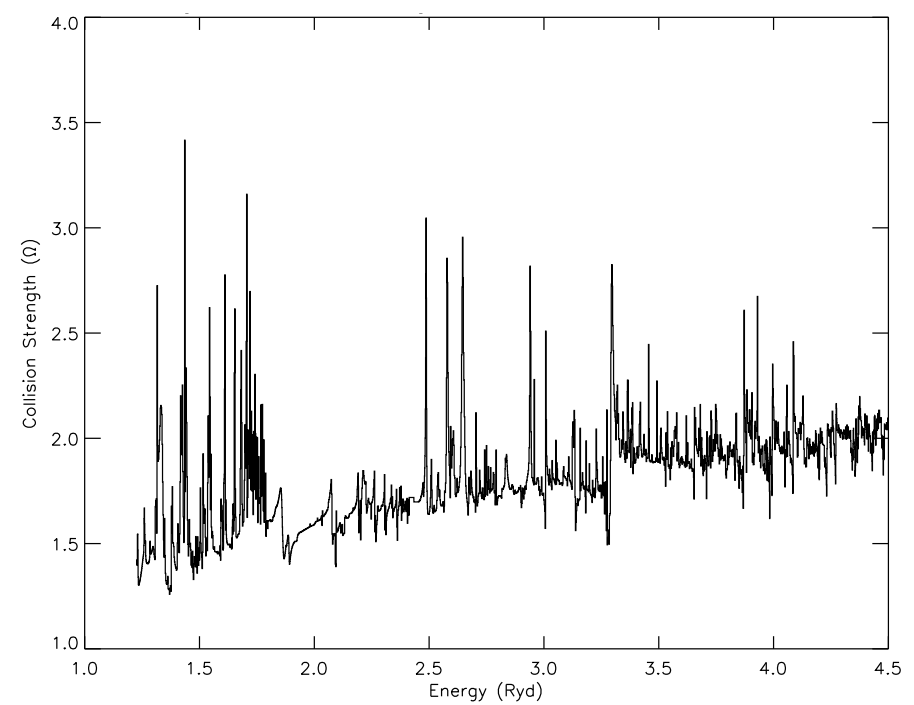

Fig. 10. Collision strengths for the $1-6\left(2 s^{2} 2 p^{2} P_{1 / 2}^{\circ}-2 s 2 p^{2}{ }^{2} D_{3 / 2}\right)$ transition of O IV.

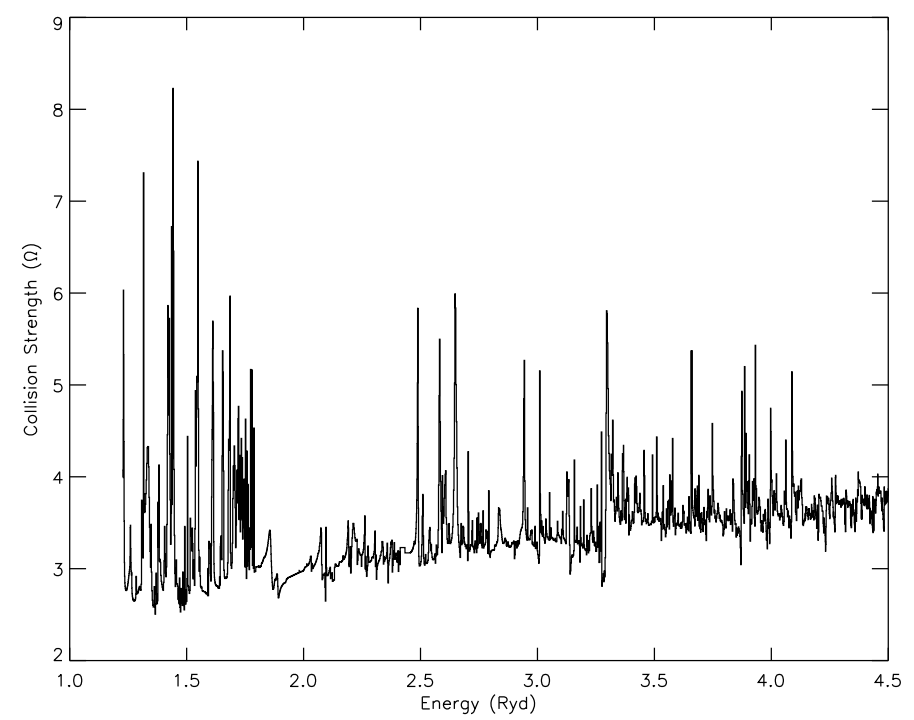

Fig. 11. Collision strengths for the $2-7\left(2 s^{2} 2 p^{2} \mathrm{P}_{3 / 2}^{\circ}-2 s 2 p^{2}{ }^{2} D_{5 / 2}\right)$ transition of O IV.

However it is clear, particularly from Figs. 12, 16 and 17, that the values of $\Upsilon$ of Blum \& Pradhan continue to increase with increasing temperature, at least for some transitions, forbidden as well as allowed, and hence are in error. This anomaly in their $\Upsilon$ values has also been noted by Tayal. Therefore, we will not discuss the results of Blum \& Pradhan any further because they must have rectified these errors when reporting the revised data in the later paper by Zhang et al. For all the transitions shown in these figures, the $\Upsilon$ by Zhang et al. are (generally) overestimated, particularly towards the lower end of the temperature range. This could be due to a variety of reasons, such as inclusion of a limited range of partial waves $(L \leq 9)$, thus overestimating the contribution of higher neglected partial waves in their top-up procedure. However, the discrepancy of their values of $\Upsilon$ with those of ours or Tayal is mainly at lower temperatures, and disappears with increasing temperature. Since the values of $\Upsilon$ of Tayal are comparatively more accurate and cover a wider range of transitions, we now focus on the comparison between our results and his. 


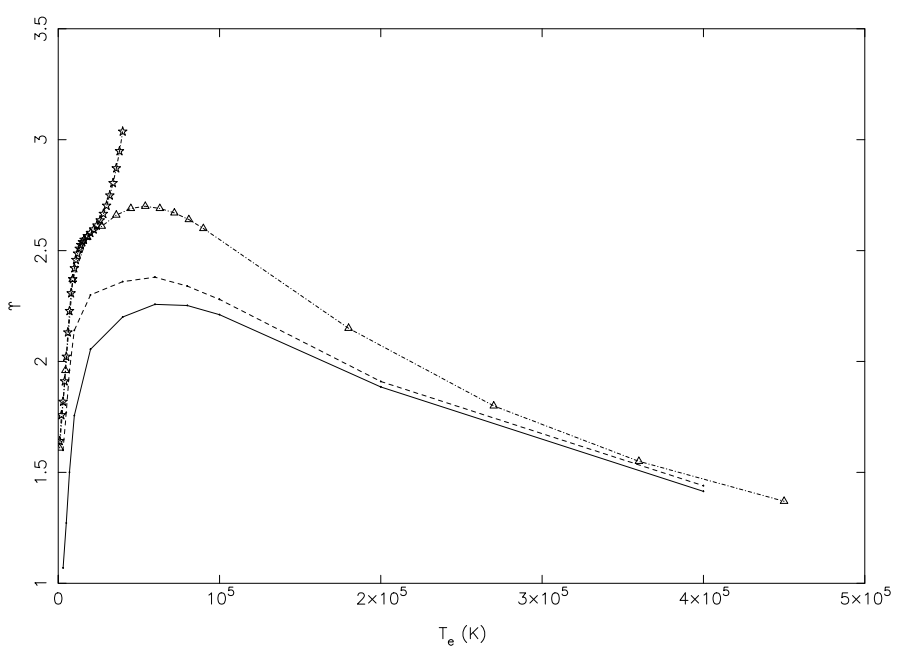

Fig. 12. Comparison of present (continuous curve) effective collision strengths with those of Blum \& Pradhan (1992: dot-dash curve with stars), Zhang et al. (1994: dot-dash curve with triangles), and Tayal (2006: broken curve) for the $1-2\left(2 \mathrm{~s}^{2} 2 \mathrm{p}^{2} \mathrm{P}_{1 / 2}^{\circ}-2 \mathrm{~s}^{2} 2 \mathrm{p}^{2} \mathrm{P}_{3 / 2}^{\circ}\right)$ transition of O IV.

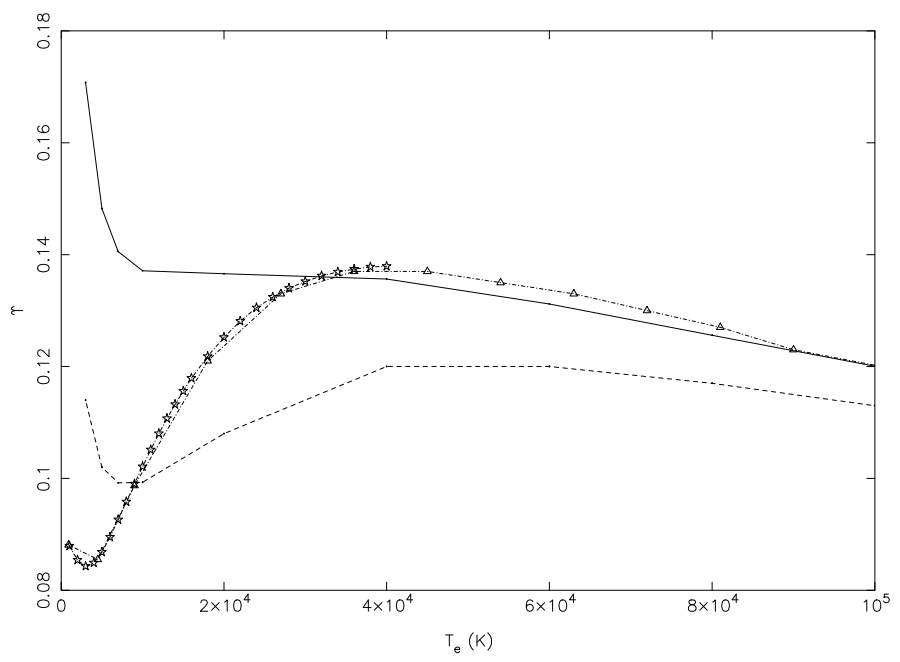

Fig. 13. Comparison of present (continuous curve) effective collision strengths with those of Blum \& Pradhan (1992: dot-dash curve with stars), Zhang et al. (1994: dot-dash curve with triangles), and Tayal (2006: broken curve) for the $2-3\left(2 \mathrm{~s}^{2} 2 \mathrm{p}^{2} \mathrm{P}_{3 / 2}^{\circ}-2 \mathrm{~s} 2 \mathrm{p}^{2}{ }^{4} \mathrm{P}_{1 / 2}\right)$ transition of O IV.

Differences between our values of $\Upsilon$ and those of Tayal (2006) are also mainly at lower temperatures, as seen in Figs. 12-17. However, the discrepancy at lower temperatures for some transitions is not only in magnitude but also in behaviour, see for example, the 2-6 transition in Fig. 16. The most likely reason for these differences in magnitude as well as behaviour is the presence (or absence) of resonances close to the threshold, as seen in Figs. 8-10. A slight shift in their placement can affect the values of $\Upsilon$ at lower temperatures, as also observed earlier for transitions in Fe XI (Aggarwal \& Keenan 2003) and Fe XIII (Aggarwal \& Keenan 2005). For example, for the 2-6 transition (not shown) we have several resonances lying close to the threshold energy. An exercise performed by removing the threshold resonances brings the two sets of $\Upsilon$ values into good agreement. However, for some other transitions, particularly the allowed ones, such as 2-3, 2-4 and 2-5 shown in Figs. 13-15, respectively, Tayal's values of $\Upsilon$ are

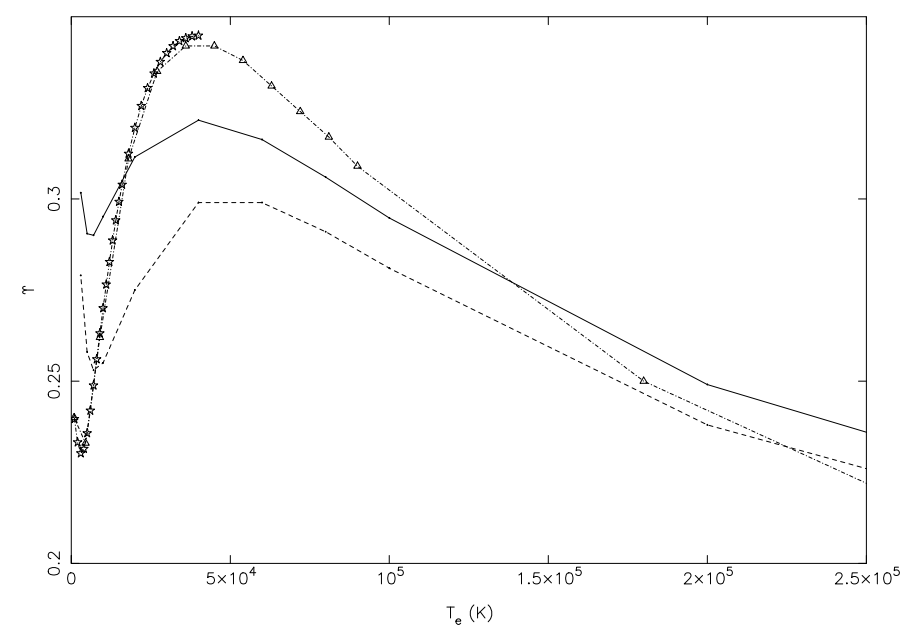

Fig. 14. Comparison of present (continuous curve) effective collision strengths with those of Blum \& Pradhan (1992: dot-dash curve with stars), Zhang et al. (1994: dot-dash curve with triangles), and Tayal (2006: broken curve) for the $2-4\left(2 s^{2} 2 p^{2} P_{3 / 2}^{\circ}-2 s 2 p^{2}{ }^{4} P_{3 / 2}\right)$ transition of O IV.

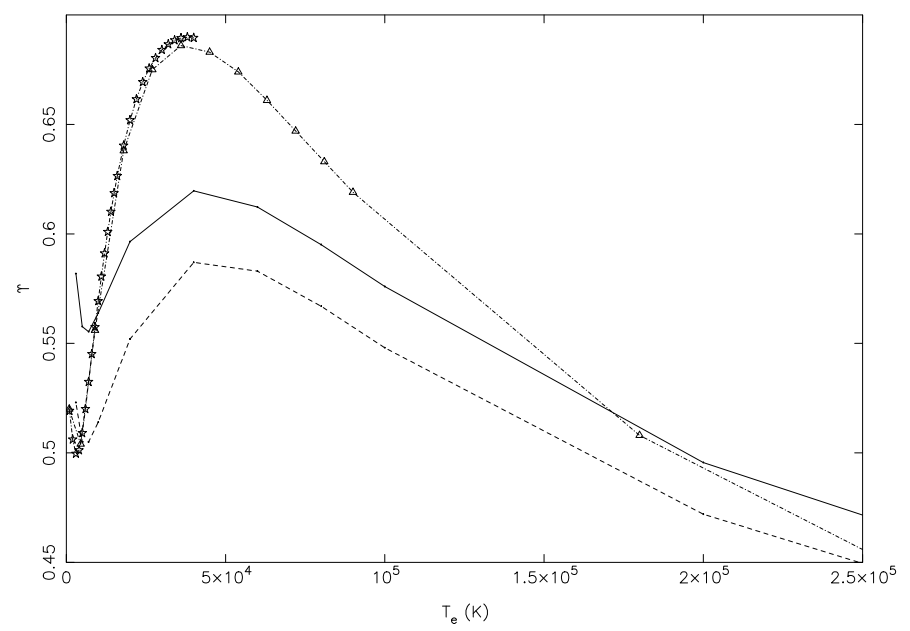

Fig. 15. Comparison of present (continuous curve) effective collision strengths with those of Blum \& Pradhan (1992: dot-dash curve with stars), Zhang et al. (1994: dot-dash curve with triangles), and Tayal (2006: broken curve) for the $2-5\left(2 \mathrm{~s}^{2} 2 \mathrm{p}^{2} \mathrm{P}_{3 / 2}^{\circ}-2 \mathrm{~s} 2 \mathrm{p}^{2}{ }^{4} \mathrm{P}_{5 / 2}\right)$ transition of O IV.

underestimated in the entire temperature range. Since Tayal has not published his values of $\Omega$ for these transitions, it is difficult to understand the differences. Furthermore, the $f$-values for these transitions are very small $\left(<10^{-7}\right)$, as seen in Table 3 . Therefore, the differences in the $\Upsilon$ values could be due to the differences in the $f$-values and subsequently the $\Omega$ values. However, there are some transitions, such as $1-19\left(2 \mathrm{~s}^{2} 2 \mathrm{p}^{2} \mathrm{P}_{1 / 2}^{\circ}-2 \mathrm{~s}^{2} 3 \mathrm{~d}^{2} \mathrm{D}_{3 / 2}\right)$ and $2-20\left(2 s^{2} 2 \mathrm{p}^{2} \mathrm{P}_{3 / 2}^{\circ}-2 \mathrm{~s}^{2} 3 \mathrm{~d}^{2} \mathrm{D}_{5 / 2}\right)$, for which the $f$-values in our calculations and those of Tayal are comparable, as shown in Table 2 . Therefore, the two sets of $\Omega$ and subsequently the $\Upsilon$ values should also be comparable. However, we notice that Tayal's results for $\Upsilon$ are overestimated by $\sim 20 \%$ in the entire temperature range as shown in Fig. 18. Both of these being allowed transitions converge slowly (see Fig. 2 for example), and therefore a larger range of partial waves as adopted in the present calculations is helpful in a more accurate determination of $\Omega$ values. Nevertheless, overall there is no (major) discrepancy between 


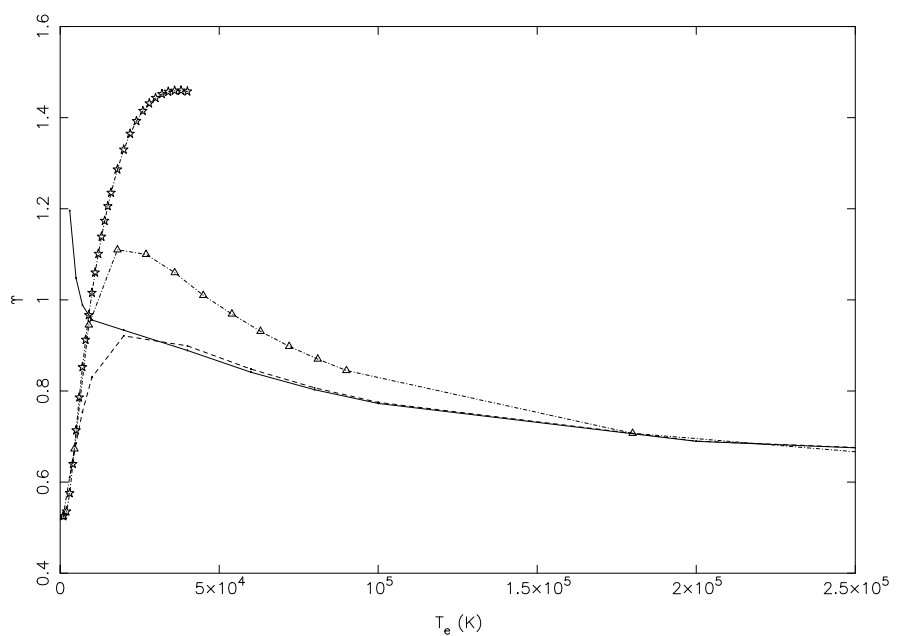

Fig. 16. Comparison of present (continuous curve) effective collision strengths with those of Blum \& Pradhan (1992: dot-dash curve with stars), Zhang et al. (1994: dot-dash curve with triangles), and Tayal (2006: broken curve) for the $2-6\left(2 s^{2} 2 p^{2} P_{3 / 2}^{\circ}-2 s 2 p^{2}{ }^{2} D_{3 / 2}\right)$ transition of O IV.

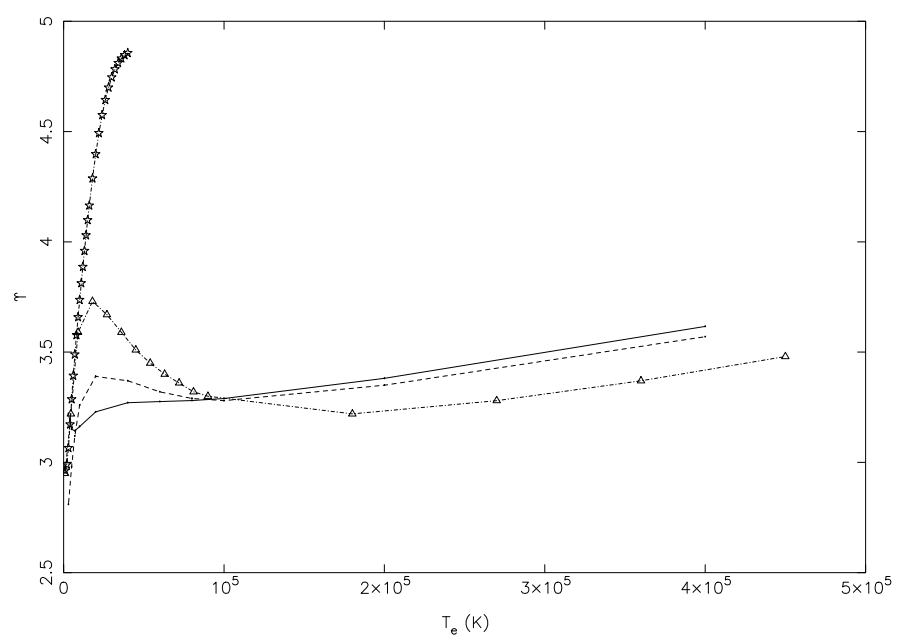

Fig. 17. Comparison of present (continuous curve) effective collision strengths with those of Blum \& Pradhan (1992: dot-dash curve with stars), Zhang et al. (1994: dot-dash curve with triangles), and Tayal (2006: broken curve) for the $2-7\left(2 \mathrm{~s}^{2} 2 \mathrm{p}^{2} \mathrm{P}_{3 / 2}^{\circ}-2 \mathrm{~s} 2 \mathrm{p}^{2}{ }^{2} \mathrm{D}_{5 / 2}\right)$ transition of O IV.

our calculations and those of Tayal, yet his results are deficient as noted earlier in Sect. 1. We elaborate on these below.

Tayal's (2006) reported data for $A$ - and $\Upsilon$ values are only for a subset of the transitions among the lowest 54 levels of $\mathrm{O}$ IV, whereas data for all transitions are required in plasma modelling. Furthermore, his reported values of $\Upsilon$ cannot be applied because of serious printing errors, as the multiplication factors of $10^{ \pm n}$ are missing from his Table 4 . For transitions such as $1-3,2-3$ and $3-9$, if one has a closer look at his results for $\Upsilon$, corrections of a factor of 100 can be applied as $\Upsilon$ should be lower towards the higher end of the temperature range. However, there are many transitions for which such corrections cannot be applied by the users, and examples include: 1-11, 1-12, 1-13, $1-14$ and $1-15$, because factors of $10^{ \pm n}$ are missing in the entire temperature range. This is clearly revealed by a comparison of his results with our values of $\Upsilon$ listed in Table 6 . Tayal's results of $\Upsilon$ for these (and many other) transitions are higher by up to three orders of magnitude, because of misprinting.

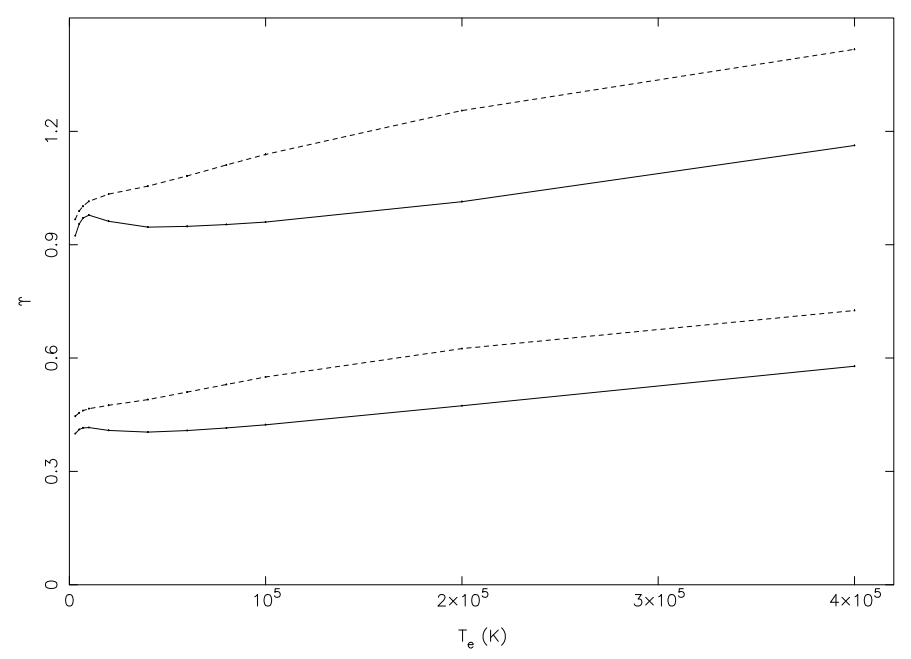

Fig. 18. Comparison of present (continuous curves) effective collision strengths with those of Tayal (2006: broken curves) for the 1-19 $\left(2 s^{2} 2 p\right.$ ${ }^{2} \mathrm{P}_{1 / 2}^{\circ}-2 \mathrm{~s}^{2} 3 \mathrm{~d}^{2} \mathrm{D}_{3 / 2}$, lower curves) and $2-20\left(2 \mathrm{~s}^{2} 2 \mathrm{p}^{2} \mathrm{P}_{3 / 2}^{\circ}-2 \mathrm{~s}^{2} 3 \mathrm{~d}^{2} \mathrm{D}_{5 / 2}\right.$, upper curves) transitions of O IV.

\section{Conclusions}

In this work we have reported energy levels and radiative rates for all transitions among the 75 levels of the $2 s^{2} 2 p, 2 s^{2} p^{2}, 2 p^{3}$, $2 \mathrm{~s}^{2} 3 \ell, 2 \mathrm{~s} 2 \mathrm{p} 3 \ell$, and $2 \mathrm{~s}^{2} 4 \ell$ configurations of $\mathrm{O}$ IV. These results have been obtained from the GRASP code, and $A$-values have been reported for four types of transitions, i.e. E1, E2, M1 and M2. The effect of extensive CI on the accuracy of the listed parameters has been fully assessed. Inclusion of CI with configurations/levels which closely interact improves the accuracy of the wavefunctions, but additional CI with higher lying levels makes an insignificant difference. Our energy levels listed in Table $1 \mathrm{~b}$ have been assessed to be accurate to better than $3 \%$, while the $A$-values are accurate to $\sim 20 \%$ for a majority of the strong transitions.

For the scattering work we have adopted the DARC code and have reported excitation rates for all transitions among the above listed 75 levels. Earlier available results of Blum \& Pradhan (1992) and Zhang et al. (1994) are limited to a few transitions, and are not assessed to be very accurate. However, there is no major discrepancy with the more recent calculations of Tayal (2006), but his results are available for only a subset of the transitions and are not easy to understand because of printing errors. Furthermore, in the present work the following improvements have been made over his calculations: (i) all 75 levels of the above configurations have been included as opposed to only 54 levels; (ii) the range of partial waves has been increased from the 25 considered by Tayal to 40 in the present work, which results in a better convergence of $\Omega$ especially at higher energies; (iii) the energy range over which $\Omega$ have been generated has been extended from 20 Ryd to 25 Ryd, which enables us to calculate values of $\Upsilon$ up to $T_{\mathrm{e}}=10^{6} \mathrm{~K}$, compared to the $T_{\mathrm{e}} \leq 4 \times 10^{5} \mathrm{~K}$ of Tayal; and finally (iv) our calculations are in $j j$ coupling which properly accounts for the relativistic effects. Through comparisons made with the earlier results, we assess that the accuracy of our values of $\Upsilon$ is better than $20 \%$. However, due to the presence of near threshold resonances, this accuracy assessment may not be correct for some transitions and for temperatures towards the lower end, particularly when there is scope for improvement in our calculated energy levels as discussed in Sect. 2. Therefore, further improvement over our results can be made by including 
levels of the $2 \mathrm{~s} 2 \mathrm{p} 4 \ell$ configurations, because these levels closely interact with the above listed 75 levels. Their inclusion in a scattering calculation will be computationally more demanding, but may be helpful in improving the accuracy of the presently reported excitation rates. However, until such calculations become available, the present results can be applied with confidence in plasma modelling.

Acknowledgements. This work has been financed by the Engineering and Physical Sciences and Science and Technology Facilities Councils of the UK, and F.P.K. is grateful to A.W.E. Aldermaston for the award of a William Penney Fellowship. We thank Dr. P. H. Norrington for providing his revised GRASP0 and DARC codes prior to publication. Finally, we thank the two Referees for their constructive criticism of our work and thus helping an overall improvement in the presentation during the process.

\section{References}

Aggarwal, K. M., \& Keenan, F. P. 2003, MNRAS, 338, 412

Aggarwal, K. M., \& Keenan, F. P. 2005, A\&A, 429, 1117

Aggarwal, K. M., \& Keenan, F. P. 2006, A\&A, 450, 1249

Aggarwal, K. M., Tayal, V., Gupta, G. P., \& Keenan, F. P. 2007, ADNDT, 93, 615

Ait-Tahar, S., Grant, I. P., \& Norrington, P. H. 1996, Phys. Rev. A, 54, 3984

Berrington, K. A., Burke, P. G., Butler, K., et al. 1987, J. Phys. B, 20, 6379

Berrington, K. A., Eissner, W. B., \& Norrington, P. H. 1995, Comput. Phys. Commun., 92, 290

Blum, R. D., \& Pradhan, A. K. 1992, ApJS, 80, 425

Corrégé, G., \& Hibbert, A. 2002, J. Phys. B, 35, 1211
Corrégé, G., \& Hibbert, A. 2004, ADNDT, 86, 19

Del Zanna, G., Berrington, K. A., \& Mason, H. E. 2004, A\&A, 422, 731

Dyall, K. G., Grant, I. P., Johnson, C. T., Parpia, F. A., \& Plummer, E. P. 1989, Comput. Phys. Commun., 55, 424

Feldman, U., Behring, W. E., Curdt, W., et al. 1997, ApJS, 113, 195

Froese-Fischer, C. 1991, Comput. Phys. Commun., 64, 369

Grant, I. P., McKenzie, B. J., Norrington, P. H., Mayers, D. F., \& Pyper, N. C. 1980, Comput. Phys. Commun., 21, 207

Gu, M. F. 2003, ApJ, 582, 1241

Harper, G. M., Jordan, C., Judge, P. G., et al. 1999, MNRAS, 303, L41

Hibbert, A. 1975a, Comput. Phys. Commun., 9, 141

Hibbert, A. 1975b, Rep. Prog. Phys., 38, 1217

Hibbert, A. 2005, Phys. Scr. T, 120, 71

Ishi, K., Suzuki, M., \& Takahashi, J. 1985, J. Phys. Soc. Japan, 54, 3742

Keenan, F. P., Ahmed, S., Brage, T., et al. 2002, MNRAS, 337, 901

Luo, D., \& Pradhan, A. K. 1990, Phys. Rev. A, 41, 165

Nahar, S. N. 1998, Phys. Rev. A, 58, 3766

Pagano, I., Linsky, J. L., Carkner, L., et al. 2000, ApJ, 532, 497

Pinnington, E. H., Irwin, D. J. G., Livingston, A. E., \& Karnahan, J. A. 1974, Can. J. Phys., 52, 1961

Pinnington, E. H., Donnely, K. E., Karnahan, J. A., \& Irwin, D. J. G. 1978, Can. J. Phys., 56, 508

Redfield, S., Linsky, J. L., Ake, T. B., et al. 2002, ApJ, 581, 626

Safronova, U. I., Johnson, W. R., \& Livingston, A. E. 1999, Phys. Rev. A, 60, 996

Smith, S. J., Lozano, J. A., Tayal, S. S., \& Chutjian, A. 2003, Phys. Rev. A, 68, 062708

Sturm, E., Lutz, D., Verma, A., et al. 2002, A\&A, 393, 821

Tachiev, G., \& Froese-Fischer, C. 2000, J. Phys. B, 33, 2419

Tayal, S. S. 2006, ApJS, 166, 634

Zhang, H. L., Graziani, M., \& Pradhan, A. K. 1994, A\&A, 283, 319 